\title{
Direct AND INVERSE SCATTERING For Selfadjoint Hamiltonian Systems on the Line
}

Tuncay Aktosun, Martin Klaus, and Cornelis van der Mee

\section{Dedicated to Israel Gohberg on the Occasion of his $70^{\text {th }}$ Birthday}

A direct and inverse scattering theory on the full line is developed for a class of first-order selfadjoint $2 n \times 2 n$ systems of differential equations with integrable potential matrices. Various properties of the corresponding scattering matrices including unitarity and their canonical Wiener-Hopf factorization are established. The Marchenko integral equations are derived and their unique solvability is proved. The unique recovery of the potential from the solutions of the Marchenko equations is shown. In the case of rational scattering matrices, state space methods are employed to construct the scattering matrix from a reflection coefficient and to recover the potential explicitly.

\section{INTRODUCTION}

Let us consider the selfadjoint Hamiltonian system of differential equations

$$
-i J_{2 n} \frac{d X(x, \lambda)}{d x}-V(x) X(x, \lambda)=\lambda X(x, \lambda), \quad x \in \mathbf{R},
$$

where

$$
J_{2 n}=\left[\begin{array}{cc}
I_{n} & 0 \\
0 & -I_{n}
\end{array}\right], \quad V(x)=\left[\begin{array}{cc}
0 & k(x) \\
k(x)^{\dagger} & 0
\end{array}\right],
$$

with $I_{n}$ the identity matrix of order $n$, the $n \times n$ matrix function $k$ has complex-valued entries belonging to $L^{1}(\mathbf{R}), \lambda \in \mathbf{R}$ is an eigenvalue parameter, and $\dagger$ denotes the matrix conjugate transpose. We call the function $k$ the potential and the parameter $\lambda$ the wavenumber. Note that $V(x)$ is a selfadjoint $2 n \times 2 n$ matrix and

$$
J_{2 n} V(x)=-V(x) J_{2 n} .
$$

We can think of $X(x, \lambda)$ in (1.1) either as a column vector of $2 n$ entries or as a $2 n \times 2 n$ matrix. For $\lambda \in \mathbf{R}$, we define the Jost solution from the left, $F_{l}(x, \lambda)$, and the Jost solution 
from the right, $F_{r}(x, \lambda)$, as the $2 n \times 2 n$ matrix solutions of (1.1) satisfying the boundary conditions

$$
\begin{aligned}
& F_{l}(x, \lambda)=e^{i \lambda J_{2 n} x}\left[I_{2 n}+o(1)\right], \quad x \rightarrow+\infty, \\
& F_{r}(x, \lambda)=e^{i \lambda J_{2 n} x}\left[I_{2 n}+o(1)\right], \quad x \rightarrow-\infty .
\end{aligned}
$$

Using (1.1), (1.3), and (1.4), we obtain

$$
\begin{aligned}
& F_{l}(x, \lambda)=e^{i \lambda J_{2 n} x}-i J_{2 n} \int_{x}^{\infty} d y e^{-i \lambda J_{2 n}(y-x)} V(y) F_{l}(y, \lambda), \\
& F_{r}(x, \lambda)=e^{i \lambda J_{2 n} x}+i J_{2 n} \int_{-\infty}^{x} d y e^{i \lambda J_{2 n}(x-y)} V(y) F_{r}(y, \lambda) .
\end{aligned}
$$

For a given square matrix function $E(x)$, let us use $\|E\|_{1}$ to denote $\int_{-\infty}^{\infty} d x\|E(x)\|$, where $\|\cdot\|$ stands for the matrix norm defined by $\|A\|=\sup _{\|v\|_{2}=1}\|A v\|_{2}$ and $\|\cdot\|_{2}$ is the Euclidean vector norm. Since the entries of $k(x)$ belong to $L^{1}(\mathbf{R})$, for each fixed $\lambda \in \mathbf{R}$ it follows that (1.5) and (1.6) are uniquely solvable and that $\left\|F_{l}(x, \lambda)\right\|$ and $\left\|F_{r}(x, \lambda)\right\|$ are bounded above by $\exp \left(\|k\|_{1}\right)$. From (1.3)-(1.6) we get

$$
\begin{aligned}
& F_{l}(x, \lambda)=e^{i \lambda J_{2 n} x}\left[a_{l}(\lambda)+o(1)\right], \quad x \rightarrow-\infty, \\
& F_{r}(x, \lambda)=e^{i \lambda J_{2 n} x}\left[a_{r}(\lambda)+o(1)\right], \quad x \rightarrow+\infty,
\end{aligned}
$$

where

$$
\begin{aligned}
& a_{l}(\lambda)=I_{2 n}-i J_{2 n} \int_{-\infty}^{\infty} d y e^{-i \lambda J_{2 n} y} V(y) F_{l}(y, \lambda), \\
& a_{r}(\lambda)=I_{2 n}+i J_{2 n} \int_{-\infty}^{\infty} d y e^{-i \lambda J_{2 n} y} V(y) F_{r}(y, \lambda) .
\end{aligned}
$$

The term "canonical differential equations" to denote the system (1.1) has been used by Melik-Adamyan [32-34], L. A. Sakhnovich [39,40], and A. L. Sakhnovich [38], who have studied the direct and inverse scattering problems for (1.1) on the half line. Under minor restrictions on the given so-called reflection function, a characterization of the scattering data corresponding to an $L^{1}$-potential on the half line was given by Melik-Adamyan [34], 
who also supplied a method to reduce the inverse spectral problem on the full line to an inverse spectral problem on the half line for a canonical equation of order $4 n$ [32]. We will comment on that characterization result at the end of Section 6. More recently, Alpay and Gohberg [3-6] have applied state space methods to derive explicit expressions for the solution of the inverse scattering problem for (1.1) on the half line from the general theory in [34] when the scattering data are rational functions and consist of either the spectral function of the differential operator $-i J_{2 n}(d / d x)-V(x)$ or a reflection function. A more self-contained treatment of these results was given by Alpay et al. [7]. Gohberg et al. have solved a similar inverse problem when the scattering data consist of the spectral function of $-i J_{2 n}(d / d x)-V(x)$ and this function is rational, both on the half line $[24,25]$ and on the full line [26].

Let us mention that there are other, more general first-order systems for which the direct and inverse scattering problems have been analyzed. Shabat [41] and Beals and Coifman $[10,11]$ considered the $n \times n$ system $d \varphi / d x=\lambda \mathbf{J} \varphi+q(x) \varphi$, where $\mathbf{J}=\operatorname{diag}\left\{\alpha_{1}, \ldots, \alpha_{n}\right\}$ with distinct complex $\alpha_{j}$ and $q(x)$ an $n \times n$ off-diagonal matrix with entries belonging to $L^{1}(\mathbf{R})$ or more restrictive classes, without requiring $q(x)$ to be selfadjoint. As indicated in [16], the distinctness of $\alpha_{j}$ is not an essential restriction. It has been proved that the inverse problem has a unique solution within a certain class of potentials for an open and dense set of scattering data. The solution of the inverse scattering problem for such linear systems is useful in solving the Cauchy problem for various nonlinear evolution equations; for details and further references, we refer the interested reader to $[2,12,16]$ and the references therein.

Note that letting $Z(x, \lambda)=\frac{1}{\sqrt{2}}\left[I_{2 n}+i \mathbf{q}_{2 n}\right] X(x, \lambda)$, where

$$
\mathbf{q}_{2 n}=\left[\begin{array}{cc}
0 & I_{n} \\
I_{n} & 0
\end{array}\right]
$$

we can convert (1.1) into the massless Dirac equation of order $2 n$ given by

$$
\frac{d Z(x, \lambda)}{d x}=\left[\begin{array}{cc}
p(x) & \lambda I_{n}-v(x) \\
-\lambda I_{n}-v(x) & -p(x)
\end{array}\right] Z(x, \lambda),
$$


where $p(x)=\frac{1}{2}\left[k(x)+k(x)^{\dagger}\right]$ and $v(x)=-\frac{1}{2} i\left[k(x)-k(x)^{\dagger}\right]$ are the real and imaginary parts of $k(x)$, respectively. The direct and inverse scattering problems for the Dirac system on the half line were studied in [22]. The interested reader is referred to [22,28,29] and the references therein for more information on the Dirac system.

The direct scattering problem for (1.1) consists of the determination of the scattering matrix $\mathbf{S}(\lambda)$ defined in (3.11) when the potential $k(x)$ is given, whereas the inverse scattering problem is the determination of $k(x)$ from $\mathbf{S}(\lambda)$ or, equivalently, from either of the reflection coefficients $R(\lambda)$ and $L(\lambda)$, which are defined in (3.7) in terms of the matrices $a_{l}(\lambda)$ and $a_{r}(\lambda)$. In this article we develop a direct and inverse scattering theory for (1.1) when $k(x)$ has entries belonging to $L^{1}(\mathbf{R})$. Working within the framework established by Faddeev [21] and Deift and Trubowitz [20] for the Schrödinger equation on the line, we derive the analyticity and asymptotic properties of the Faddeev matrices and the scattering coefficients, employ them to derive a Riemann-Hilbert problem and various Marchenko integral equations, and recover the potential in terms of the solutions of the Marchenko equations. We derive the unitarity of the scattering matrix and exploit this property to prove the unique solvability of the Marchenko equations. We also establish the unique canonical Wiener-Hopf factorization of the (unitarily-dilated) scattering matrix and show how the potential is obtained once the factors in the factorization are known. We then give a rather general sufficient condition on the reflection coefficient to lead to a potential whose entries belong to $L^{1}(\mathbf{R})$. After that, for rational reflection coefficients we present a procedure to compute explicitly the scattering matrix from a reflection coefficient. This is no longer as elementary as in the case of the Schrödinger equation $[20,21]$ and involves a suitable extension of a contractive $n \times n$ matrix function to a unitary $2 n \times 2 n$ matrix function (cf. $[8,27]$ ). We then apply state space methods [13] to solve the Marchenko equations and the inverse problem explicitly when the reflection coefficients are rational. This approach provides us with a systematic inversion method for rational reflection coefficients which is more complete and totally different from previous methods, for example, those 
used in [9].

This article is organized as follows. In Section 2 we introduce the Faddeev matrices, obtain their analyticity properties, and analyze some other properties of the Faddeev matrices and the Jost solutions of (1.1). In Section 3 we define the scattering matrix $\mathbf{S}(\lambda)$ in terms of the spatial asymptotics of the Jost solutions, prove the unitarity of $\mathbf{S}(\lambda)$, and obtain various properties of the scattering coefficients. In Section 4 we analyze the Fourier transforms of the Faddeev matrices and the scattering coefficients. We then go on, in Section 5, to derive a Riemann-Hilbert problem for the Faddeev matrices and show that the (unitarily-dilated) scattering matrix has a canonical Wiener-Hopf factorization; we also show by an example that the canonical factorization of the scattering matrix and the unique recovery of the potential need no longer be true if the entries of the potential do not belong to $L^{1}(\mathbf{R})$. In Section 6, we convert the Riemann-Hilbert problem into both coupled and uncoupled Marchenko integral equations, prove their unique solvability by a contraction mapping argument, and give a partial characterization of the scattering data corresponding to potentials with entries in $L^{1}(\mathbf{R})$. In Section 7 we show how the scattering matrix can be constructed from a reflection coefficient, and we also construct $\mathbf{S}(\lambda)$ explicitly when one of the reflection coefficients is a rational function. Finally, in Section 8 we give an explicit solution of the inverse scattering problem with rational reflection coefficients; this is done by using the minimal realization of the reflection coefficients as input to the Marchenko equations.

\section{Scattering Solutions}

In this section we introduce the Faddeev matrices and study their properties. The results obtained here will be used later to establish various properties of the scattering matrix and to solve the inverse scattering problem by the Marchenko method.

Proposition 2.1. Let $X(x, \lambda)$ and $Y(x, \lambda)$ be any two solutions of (1.1). Then, for real $\lambda, X(x, \lambda)^{\dagger} J_{2 n} Y(x, \lambda)$ is independent of $x$. 
Proof. This result follows from (1.1) using the selfadjointness of $V(x)$ and $J_{2 n}$.

Proposition 2.2. For $\lambda \in \mathbf{R}$, either of the Jost solutions $F_{l}(x, \lambda)$ and $F_{r}(x, \lambda)$ forms a fundamental matrix of (1.1) and has determinant equal to one, and

$$
\operatorname{det} a_{l}(\lambda)=\operatorname{det} a_{r}(\lambda)=1
$$

Moreover, for $\lambda \in \mathbf{R}$, the Jost solutions satisfy

$$
\begin{gathered}
F_{l}(x, \lambda)=F_{r}(x, \lambda) a_{l}(\lambda), \\
F_{r}(x, \lambda)^{\dagger} J_{2 n} F_{l}(x, \lambda)=a_{r}(\lambda)^{\dagger} J_{2 n}=J_{2 n} a_{l}(\lambda), \\
F_{l}(x, \lambda)^{\dagger} J_{2 n} F_{l}(x, \lambda)=a_{l}(\lambda)^{\dagger} J_{2 n} a_{l}(\lambda)=J_{2 n}, \\
F_{r}(x, \lambda)^{\dagger} J_{2 n} F_{r}(x, \lambda)=a_{r}(\lambda)^{\dagger} J_{2 n} a_{r}(\lambda)=J_{2 n},
\end{gathered}
$$

and hence

$$
\begin{gathered}
a_{l}(\lambda) a_{r}(\lambda)=a_{r}(\lambda) a_{l}(\lambda)=I_{2 n} \\
a_{l}(\lambda)^{-1}=J_{2 n} a_{l}(\lambda)^{\dagger} J_{2 n}, \quad a_{r}(\lambda)^{-1}=J_{2 n} a_{r}(\lambda)^{\dagger} J_{2 n} .
\end{gathered}
$$

Proof. From (1.1) it follows [35] that

$$
\frac{d\left[\operatorname{det} F_{l}(x, \lambda)\right]}{d x}=\left(\operatorname{tr}\left\{i J_{2 n} V(x)+i \lambda J_{2 n}\right\}\right)\left(\operatorname{det} F_{l}(x, \lambda)\right)
$$

where tr denotes the matrix trace. By $(1.2), i J_{2 n} V(x)+i \lambda J_{2 n}$ has zero trace, and hence $\operatorname{det} F_{l}(x, \lambda)$ is independent of $x$ and its value can be evaluated as $x \rightarrow+\infty$. Thus, we get $\operatorname{det} F_{l}(x, \lambda)=1$, from which we also conclude that $F_{l}(x, \lambda)$ is a fundamental matrix of (1.1). Similarly, we find that $\operatorname{det} F_{r}(x, \lambda)=1$ and $F_{r}(x, \lambda)$ is a fundamental matrix of (1.1). Then, from (1.2), (1.7), and (1.8) we obtain (2.1). Since either of $F_{l}(x, \lambda)$ and $F_{r}(x, \lambda)$ is a fundamental matrix of (1.1), with the help of (1.3) and (1.7), we get (2.2). Using Proposition 2.1, we obtain (2.3)-(2.5) by evaluating $F_{r}(x, \lambda)^{\dagger} J_{2 n} F_{l}(x, \lambda)$, 
$F_{l}(x, \lambda)^{\dagger} J_{2 n} F_{l}(x, \lambda)$, and $F_{r}(x, \lambda)^{\dagger} J_{2 n} F_{r}(x, \lambda)$ as $x \rightarrow \pm \infty$. Equations (2.6) and (2.7) then follow readily.

In terms of the Jost solutions, the Faddeev matrices $M_{l}(x, \lambda)$ and $M_{r}(x, \lambda)$ are defined as

$$
M_{l}(x, \lambda)=F_{l}(x, \lambda) e^{-i \lambda J_{2 n} x}, \quad M_{r}(x, \lambda)=F_{r}(x, \lambda) e^{-i \lambda J_{2 n} x}
$$

From (1.3) and (1.4) we see that

$$
\begin{aligned}
& M_{l}(x, \lambda)=I_{2 n}+o(1), \quad x \rightarrow+\infty \\
& M_{r}(x, \lambda)=I_{2 n}+o(1), \quad x \rightarrow-\infty .
\end{aligned}
$$

Let us partition the Jost solutions and Faddeev matrices into $n \times n$ matrices as follows:

$$
\begin{aligned}
& F_{l}(x, \lambda)=\left[\begin{array}{ll}
F_{l 1}(x, \lambda) & F_{l 2}(x, \lambda) \\
F_{l 3}(x, \lambda) & F_{l 4}(x, \lambda)
\end{array}\right], \quad F_{r}(x, \lambda)=\left[\begin{array}{ll}
F_{r 1}(x, \lambda) & F_{r 2}(x, \lambda) \\
F_{r 3}(x, \lambda) & F_{r 4}(x, \lambda)
\end{array}\right], \\
& M_{l}(x, \lambda)=\left[\begin{array}{ll}
M_{l 1}(x, \lambda) & M_{l 2}(x, \lambda) \\
M_{l 3}(x, \lambda) & M_{l 4}(x, \lambda)
\end{array}\right], \quad M_{r}(x, \lambda)=\left[\begin{array}{ll}
M_{r 1}(x, \lambda) & M_{r 2}(x, \lambda) \\
M_{r 3}(x, \lambda) & M_{r 4}(x, \lambda)
\end{array}\right] \text {. }
\end{aligned}
$$

By $\mathbf{C}^{+}$and $\mathbf{C}^{-}$we denote the open upper half and lower half complex plane, respectively. We also define

$$
\sigma_{ \pm}(x)= \pm \int_{x}^{ \pm \infty} d y\|k(y)\|
$$

Proposition 2.3. Assume that the entries of $k(x)$ belong to $L^{1}(\mathbf{R})$. Then:

(i) For each fixed $x \in \mathbf{R},\left[\begin{array}{l}M_{l 1}(x, \lambda) \\ M_{l 3}(x, \lambda)\end{array}\right]$ can be extended to a matrix function that is continuous in $\lambda \in \overline{\mathbf{C}^{+}}$and analytic in $\lambda \in \mathbf{C}^{+}$and tends to $\left[\begin{array}{c}I_{n} \\ 0\end{array}\right]$ as $\lambda \rightarrow \infty$ in $\overline{\mathbf{C}^{+}}$.

(ii) For all $\lambda \in \overline{\mathbf{C}^{+}}$, each entry of $M_{l 1}(x, \lambda)$ and $M_{l 3}(x, \lambda)$ is bounded by $e^{\sigma_{+}(x)}$ in absolute value.

(iii) For each fixed $x \in \mathbf{R},\left[\begin{array}{l}M_{l 2}(x, \lambda) \\ M_{l 4}(x, \lambda)\end{array}\right]$ can be extended to a matrix function that is continuous in $\lambda \in \overline{\mathbf{C}^{-}}$and analytic in $\lambda \in \mathbf{C}^{-}$and tends to $\left[\begin{array}{c}0 \\ I_{n}\end{array}\right]$ as $\lambda \rightarrow \infty$ in $\overline{\mathbf{C}^{-}}$. 
(iv) For all $\lambda \in \overline{\mathbf{C}^{-}}$, each entry of $M_{l 2}(x, \lambda)$ and $M_{l 4}(x, \lambda)$ is bounded by $e^{\sigma_{+}(x)}$ in absolute value.

Proof. Using (2.8) in (1.5), we obtain

$$
M_{l}(x, \lambda)=I_{2 n}-i J_{2 n} \int_{x}^{\infty} d y e^{-i \lambda J_{2 n}(y-x)} V(y) M_{l}(y, \lambda) e^{i \lambda J_{2 n}(y-x)}
$$

Iterating (2.12) once, we get the uncoupled systems

$$
\begin{gathered}
M_{l 1}(x, \lambda)=I_{n}+\int_{x}^{\infty} d y \int_{y}^{\infty} d z e^{2 i \lambda(z-y)} k(y) k(z)^{\dagger} M_{l 1}(z, \lambda), \\
M_{l 2}(x, \lambda)=-i \int_{x}^{\infty} d y e^{-2 i \lambda(y-x)} k(y)+\int_{x}^{\infty} d y \int_{y}^{\infty} d z e^{-2 i \lambda(y-x)} k(y) k(z)^{\dagger} M_{l 2}(z, \lambda), \\
M_{l 3}(x, \lambda)=i \int_{x}^{\infty} d y e^{2 i \lambda(y-x)} k(y)^{\dagger}+\int_{x}^{\infty} d y \int_{y}^{\infty} d z e^{2 i \lambda(y-x)} k(y)^{\dagger} k(z) M_{l 3}(z, \lambda), \\
M_{l 4}(x, \lambda)=I_{n}+\int_{x}^{\infty} d y \int_{y}^{\infty} d z e^{-2 i \lambda(z-y)} k(y)^{\dagger} k(z) M_{l 4}(z, \lambda) .
\end{gathered}
$$

Iterating the Volterra integral equations (2.13) and (2.15), we prove that the series of iterates converge absolutely and uniformly in $\lambda \in \overline{\mathbf{C}^{+}}$, and we also get the estimate in (ii). Similarly, we prove that the series of iterates of (2.14) and (2.16) converge absolutely and uniformly in $\lambda \in \overline{\mathbf{C}^{-}}$and that the estimate in (iv) holds. The large- $\lambda$ asymptotics follow from the use of the Riemann-Lebesgue lemma in (2.13)-(2.16).

As in Proposition 2.3, we have a similar result for the Faddeev matrix $M_{r}(x, \lambda)$ :

Proposition 2.4. Assume that the entries of $k(x)$ belong to $L^{1}(\mathbf{R})$. Then:

(i) For each fixed $x \in \mathbf{R},\left[\begin{array}{l}M_{r 1}(x, \lambda) \\ M_{r 3}(x, \lambda)\end{array}\right]$ can be extended to a matrix function that is continuous in $\lambda \in \overline{\mathbf{C}^{-}}$and analytic in $\lambda \in \mathbf{C}^{-}$and tends to $\left[\begin{array}{c}I_{n} \\ 0\end{array}\right]$ as $\lambda \rightarrow \infty$ in $\overline{\mathbf{C}^{-}}$.

(ii) For all $\lambda \in \overline{\mathbf{C}^{-}}$, each entry of $M_{r 1}(x, \lambda)$ and $M_{r 3}(x, \lambda)$ is bounded by $e^{\sigma_{-}(x)}$ in absolute value.

(iii) For each fixed $x \in \mathbf{R},\left[\begin{array}{l}M_{r 2}(x, \lambda) \\ M_{r 4}(x, \lambda)\end{array}\right]$ can be extended to a matrix function that is continuous in $\lambda \in \overline{\mathbf{C}^{+}}$and analytic in $\lambda \in \mathbf{C}^{+}$and tends to $\left[\begin{array}{c}0 \\ I_{n}\end{array}\right]$ as $\lambda \rightarrow \infty$ in $\overline{\mathbf{C}^{+}}$. 
(iv) For all $\lambda \in \overline{\mathbf{C}^{+}}$, each entry of $M_{r 2}(x, \lambda)$ and $M_{r 4}(x, \lambda)$ is bounded by $e^{\sigma_{-}(x)}$ in absolute value.

Proof. Using (2.8) in (1.6), we obtain

$$
M_{r}(x, \lambda)=I_{2 n}+i J_{2 n} \int_{-\infty}^{x} d y e^{i \lambda J_{2 n}(x-y)} V(y) M_{r}(y, \lambda) e^{-i \lambda J_{2 n}(x-y)}
$$

Iterating (2.17) once we obtain the four systems given by

$$
\begin{gathered}
M_{r 1}(x, \lambda)=I_{n}+\int_{-\infty}^{x} d y \int_{-\infty}^{y} d z e^{-2 i \lambda(y-z)} k(y) k(z)^{\dagger} M_{r 1}(z, \lambda), \\
M_{r 2}(x, \lambda)=i \int_{-\infty}^{x} d y e^{2 i \lambda(x-y)} k(y)+\int_{-\infty}^{x} d y \int_{-\infty}^{y} d z e^{2 i \lambda(x-y)} k(y) k(z)^{\dagger} M_{r 2}(z, \lambda), \\
M_{r 3}(x, \lambda)=-i \int_{-\infty}^{x} d y e^{-2 i \lambda(x-y)} k(y)^{\dagger}+\int_{-\infty}^{x} d y \int_{-\infty}^{y} d z e^{-2 i \lambda(x-y)} k(y)^{\dagger} k(z) M_{r 3}(z, \lambda), \\
M_{r 4}(x, \lambda)=I_{n}+\int_{-\infty}^{x} d y \int_{-\infty}^{y} d z e^{2 i \lambda(y-z)} k(y)^{\dagger} k(z) M_{r 4}(z, \lambda) .
\end{gathered}
$$

Iterating (2.18)-(2.21) as in the proof of Proposition 2.3, we complete the proof.

Let us write

$$
a_{l}(\lambda)=\left[\begin{array}{ll}
a_{l 1}(\lambda) & a_{l 2}(\lambda) \\
a_{l 3}(\lambda) & a_{l 4}(\lambda)
\end{array}\right], \quad a_{r}(\lambda)=\left[\begin{array}{ll}
a_{r 1}(\lambda) & a_{r 2}(\lambda) \\
a_{r 3}(\lambda) & a_{r 4}(\lambda)
\end{array}\right]
$$

From $(1.7),(1.8)$, and (2.8) we see that

$$
\begin{gathered}
{\left[\begin{array}{ll}
a_{l 1}(\lambda) & a_{l 2}(\lambda) \\
a_{l 3}(\lambda) & a_{l 4}(\lambda)
\end{array}\right]=\lim _{x \rightarrow-\infty}\left[\begin{array}{cc}
M_{l 1}(x, \lambda) & e^{-2 i \lambda x} M_{l 2}(x, \lambda) \\
e^{2 i \lambda x} M_{l 3}(x, \lambda) & M_{l 4}(x, \lambda)
\end{array}\right],} \\
{\left[\begin{array}{ll}
a_{r 1}(\lambda) & a_{r 2}(\lambda) \\
a_{r 3}(\lambda) & a_{r 4}(\lambda)
\end{array}\right]=\lim _{x \rightarrow+\infty}\left[\begin{array}{cc}
M_{r 1}(x, \lambda) & e^{-2 i \lambda x} M_{r 2}(x, \lambda) \\
e^{2 i \lambda x} M_{r 3}(x, \lambda) & M_{r 4}(x, \lambda)
\end{array}\right] .}
\end{gathered}
$$

Using (2.12), (2.17), (2.23), and (2.24) we find the integral representations

$$
a_{l 1}(\lambda)=I_{n}-i \int_{-\infty}^{\infty} d y k(y) M_{l 3}(y, \lambda)
$$




$$
\begin{aligned}
& a_{l 2}(\lambda)=-i \int_{-\infty}^{\infty} d y e^{-2 i \lambda y} k(y) M_{l 4}(y, \lambda), \\
& a_{l 3}(\lambda)=i \int_{-\infty}^{\infty} d y e^{2 i \lambda y} k(y)^{\dagger} M_{l 1}(y, \lambda), \\
& a_{l 4}(\lambda)=I_{n}+i \int_{-\infty}^{\infty} d y k(y)^{\dagger} M_{l 2}(y, \lambda), \\
& a_{r 1}(\lambda)=I_{n}+i \int_{-\infty}^{\infty} d y k(y) M_{r 3}(y, \lambda), \\
& a_{r 2}(\lambda)=i \int_{-\infty}^{\infty} d y e^{-2 i \lambda y} k(y) M_{r 4}(y, \lambda), \\
& a_{r 3}(\lambda)=-i \int_{-\infty}^{\infty} d y e^{2 i \lambda y} k(y)^{\dagger} M_{r 1}(y, \lambda), \\
& a_{r 4}(\lambda)=I_{n}-i \int_{-\infty}^{\infty} d y k(y)^{\dagger} M_{r 2}(y, \lambda) .
\end{aligned}
$$

Proposition 2.5. Assume that the entries of $k(x)$ belong to $L^{1}(\mathbf{R})$. Then:

(i) The matrices $a_{l 1}(\lambda)$ and $a_{r 4}(\lambda)$ are continuous in $\lambda \in \overline{\mathbf{C}^{+}}$and analytic in $\lambda \in \mathbf{C}^{+}$ and tend to $I_{n}$ as $\lambda \rightarrow \infty$ in $\overline{\mathbf{C}^{+}}$.

(ii) The matrices $a_{l 4}(\lambda)$ and $a_{r 1}(\lambda)$ are continuous in $\lambda \in \overline{\mathbf{C}^{-}}$and analytic in $\lambda \in \mathbf{C}^{-}$ and tend to $I_{n}$ as $\lambda \rightarrow \infty$ in $\overline{\mathbf{C}^{-}}$.

(iii) The matrices $a_{l 2}(\lambda), a_{l 3}(\lambda), a_{r 2}(\lambda)$, and $a_{r 3}(\lambda)$ are continuous in $\lambda \in \mathbf{R}$ and vanish as $\lambda \rightarrow \pm \infty$.

(iv) The matrices $a_{l 2}(\lambda), a_{l 3}(\lambda), a_{r 2}(\lambda)$, and $a_{r 3}(\lambda)$ satisfy

$$
a_{r 2}(\lambda)=-a_{l 3}(\lambda)^{\dagger}, \quad a_{r 3}(\lambda)=-a_{l 2}(\lambda)^{\dagger}, \quad \lambda \in \mathbf{R}
$$

Proof. Using Propositions 2.3 and 2.4 in (2.25)-(2.32), we get (i), (ii), and (iii). We obtain (iv) from (2.3).

Using the notations of (2.9), let us form the following matrices:

$$
f_{+}(x, \lambda)=\left[\begin{array}{ll}
F_{l 1}(x, \lambda) & F_{r 2}(x, \lambda) \\
F_{l 3}(x, \lambda) & F_{r 4}(x, \lambda)
\end{array}\right], \quad f_{-}(x, \lambda)=\left[\begin{array}{ll}
F_{r 1}(x, \lambda) & F_{l 2}(x, \lambda) \\
F_{r 3}(x, \lambda) & F_{l 4}(x, \lambda)
\end{array}\right] .
$$


Let an asterisk denote complex conjugation. From Propositions 2.3 and 2.4 , it follows that $f_{+}(x, \lambda)$ is a solution of (1.1) that is continuous in $\lambda \in \overline{\mathbf{C}^{+}}$and analytic in $\lambda \in \mathbf{C}^{+}$; similarly, $f_{-}(x, \lambda)$ is a solution of $(1.1)$ that is continuous in $\lambda \in \overline{\mathbf{C}^{-}}$and analytic in $\lambda \in \mathbf{C}^{-}$.

Proposition 2.6. The $2 n \times 2 n$ matrix $f_{-}\left(x, \lambda^{*}\right)^{\dagger} J_{2 n} f_{+}(x, \lambda)$ is independent of $x$ for all $\lambda \in \overline{\mathbf{C}^{+}}$. Similarly, $f_{+}\left(x, \lambda^{*}\right)^{\dagger} J_{2 n} f_{-}(x, \lambda)$ is independent of $x$ for all $\lambda \in \overline{\mathbf{C}^{-}}$. We have

$$
f_{-}\left(x, \lambda^{*}\right)^{\dagger} J_{2 n} f_{+}(x, \lambda)=\left[\begin{array}{cc}
a_{l 1}(\lambda) & 0 \\
0 & -a_{r 4}(\lambda)
\end{array}\right], \quad \lambda \in \overline{\mathbf{C}^{+}} .
$$

Further, $a_{l 1}(\lambda)^{\dagger}$ and $a_{r 4}(\lambda)^{\dagger}$ have analytic extensions to $\mathbf{C}^{-}, a_{r 1}(\lambda)^{\dagger}$ and $a_{l 4}(\lambda)^{\dagger}$ have analytic extensions to $\mathbf{C}^{+}$, and

$$
\begin{array}{lll}
a_{l 1}(\lambda)=a_{r 1}\left(\lambda^{*}\right)^{\dagger}, & a_{r 4}(\lambda)=a_{l 4}\left(\lambda^{*}\right)^{\dagger}, & \lambda \in \overline{\mathbf{C}^{+}}, \\
a_{r 1}(\lambda)=a_{l 1}\left(\lambda^{*}\right)^{\dagger}, & a_{l 4}(\lambda)=a_{r 4}\left(\lambda^{*}\right)^{\dagger}, & \lambda \in \overline{\mathbf{C}^{-}} .
\end{array}
$$

Proof. Using (1.1), one can show that the $x$-derivative of $f_{\mp}\left(x, \lambda^{*}\right)^{\dagger} J_{2 n} f_{ \pm}(x, \lambda)$ vanishes for $\lambda \in \overline{\mathbf{C}^{ \pm}}$, and evaluating it as $x \rightarrow \pm \infty$ we get (2.35)-(2.37).

Proposition 2.7. For $\lambda \in \mathbf{R}$, either of $f_{+}(x, \lambda)$ and $f_{-}(x, \lambda)$ is nonsingular and hence is a fundamental matrix for (1.1).

Proof. As in the proof of Proposition 2.1 we find that $\operatorname{det} f_{+}(x, \lambda)$ is independent of $x$, and evaluating that determinant as $x \rightarrow \pm \infty$ we obtain

$$
\operatorname{det} f_{+}(x, \lambda)=\operatorname{det} a_{l 1}(\lambda)=\operatorname{det} a_{r 4}(\lambda)
$$

From (2.3) it follows that

$$
a_{l 1}(\lambda)^{\dagger} a_{l 1}(\lambda)=I_{n}+a_{l 3}(\lambda)^{\dagger} a_{l 3}(\lambda), \quad \lambda \in \mathbf{R}
$$

and hence $a_{l 1}(\lambda)$ is invertible for all $\lambda \in \mathbf{R}$. Thus, $f_{+}(x, \lambda)$ is nonsingular and forms a fundamental matrix for (1.1). Similarly, we get

$$
\operatorname{det} f_{-}(x, \lambda)=\operatorname{det} a_{r 1}(\lambda)=\operatorname{det} a_{l 4}(\lambda),
$$


and hence with the help of (2.36) and (2.39), we conclude that $f_{-}(x, \lambda)$ is nonsingular and forms a fundamental matrix for (1.1).

Next we will prove that $f_{+}(x, \lambda)$ is nonsingular for $\lambda \in \overline{\mathbf{C}^{+}}$and $f_{-}(x, \lambda)$ is nonsingular for $\lambda \in \overline{\mathbf{C}^{-}}$. First, using (2.10) and (2.34), let us define

$$
\begin{aligned}
& m_{+}(x, \lambda)=\left[\begin{array}{ll}
M_{l 1}(x, \lambda) & M_{r 2}(x, \lambda) \\
M_{l 3}(x, \lambda) & M_{r 4}(x, \lambda)
\end{array}\right]=f_{+}(x, \lambda) e^{-i \lambda J_{2 n} x}, \\
& m_{-}(x, \lambda)=\left[\begin{array}{ll}
M_{r 1}(x, \lambda) & M_{l 2}(x, \lambda) \\
M_{r 3}(x, \lambda) & M_{l 4}(x, \lambda)
\end{array}\right]=f_{-}(x, \lambda) e^{-i \lambda J_{2 n} x} .
\end{aligned}
$$

Proposition 2.8. For each $\lambda \in \overline{\mathbf{C}^{+}}, f_{+}(x, \lambda)$ is a fundamental matrix for (1.1). Similarly, for each $\lambda \in \overline{\mathbf{C}^{-}}, f_{-}(x, \lambda)$ is a fundamental matrix for (1.1).

Proof. The proof for $f_{-}(x, \lambda)$ is similar to the proof for $f_{+}(x, \lambda)$, and hence we will only present the latter. From Proposition 2.7, we already know that $f_{+}(x, \lambda)$ is a fundamental matrix for (1.1) when $\lambda \in \mathbf{R}$. Thus, we only need to prove that the columns of $f_{+}(x, \lambda)$ are linearly independent for $\lambda \in \mathbf{C}^{+}$. Because of (1.3) and (1.4), the first $n$ columns of $f_{+}(x, \lambda)$ are linearly independent for $\lambda \in \mathbf{C}^{+}$, and also the last $n$ columns of $f_{+}(x, \lambda)$ are linearly independent for $\lambda \in \mathbf{C}^{+}$. It is sufficient to prove that an arbitrary nontrivial linear combination of the first $n$ columns cannot be written as a linear combination of the last $n$ columns of $f_{+}(x, \lambda)$. Otherwise, we would have

$$
\sum_{s=1}^{n} c_{s}(\lambda) e^{i \lambda x} C_{s}(x, \lambda)=\sum_{q=n+1}^{2 n} c_{q}(\lambda) e^{-i \lambda x} C_{q}(x, \lambda)
$$

where $C_{j}(x, \lambda)$ represents the $j$ th column of the matrix $m_{+}(x, \lambda)$ defined in $(2.41)$, and $c_{j}(\lambda)$ are independent of $x$. From Proposition 2.3 (ii) and Proposition 2.4 (ii), it follows that each entry of $C_{j}(x, \lambda)$ is uniformly bounded in $x \in \mathbf{R}$ for each $\lambda \in \mathbf{C}^{+}$. The lefthand side in (2.43) decreases exponentially as $x \rightarrow+\infty$ while the right-hand side decreases exponentially as $x \rightarrow-\infty$; this would turn either side into a nontrivial $L^{2}$-solution of (1.1), 
which is a contradiction because the selfadjoint differential operator of (1.1) cannot have nonreal eigenvalues.

When $\operatorname{Im} \lambda \neq 0$, a result similar to the one in Proposition 2.8 was proved in [30].

From Propositions 2.3, 2.4, and 2.8 we obtain the following result.

COROLlaRY 2.9. For each $x \in \mathbf{R}$, the $2 n \times 2 n$ matrix $m_{+}(x, \lambda)$ and its inverse $m_{+}(x, \lambda)^{-1}$ are continuous in $\lambda \in \overline{\mathbf{C}^{+}}$, are analytic in $\lambda \in \mathbf{C}^{+}$, and converge to $I_{2 n}$ as $\lambda \rightarrow \infty$ in $\overline{\mathbf{C}^{+}}$. Similarly, for each $x \in \mathbf{R}$, the $2 n \times 2 n$ matrix $m_{-}(x, \lambda)$ and its inverse $m_{-}(x, \lambda)^{-1}$ are continuous in $\lambda \in \overline{\mathbf{C}^{-}}$, are analytic in $\lambda \in \mathbf{C}^{-}$, and converge to $I_{2 n}$ as $\lambda \rightarrow \infty$ in $\overline{\mathbf{C}^{-}}$.

Proposition 2.10. Assume that the entries of $k(x)$ belong to $L^{1}(\mathbf{R})$. Then:

(i) The matrices $a_{l 1}(\lambda)$ and $a_{r 4}(\lambda)$ are invertible for each $\lambda \in \overline{\mathbf{C}^{+}}$, and $a_{l 4}(\lambda)$ and $a_{r 1}(\lambda)$ are invertible for each $\lambda \in \overline{\mathbf{C}^{-}}$.

(ii) The matrix functions $a_{l 1}(\lambda)^{-1}$ and $a_{r 4}(\lambda)^{-1}$ are continuous in $\overline{\mathbf{C}^{+}}$and analytic in $\mathbf{C}^{+}$and tend to $I_{n}$ as $\lambda \rightarrow \infty$ in $\overline{\mathbf{C}^{+}}$.

(iii) The matrix functions $a_{l 4}(\lambda)^{-1}$ and $a_{r 1}(\lambda)^{-1}$ are continuous in $\overline{\mathbf{C}^{-}}$and analytic in $\mathbf{C}^{-}$and tend to $I_{n}$ as $\lambda \rightarrow \infty$ in $\overline{\mathbf{C}^{-}}$.

Proof. From Proposition 2.8 it follows that $f_{+}(x, \lambda)$ and $f_{-}\left(x, \lambda^{*}\right)^{\dagger}$ are nonsingular for $\lambda \in \overline{\mathbf{C}^{+}}$. Hence, (2.35) implies that $a_{l 1}(\lambda)$ and $a_{r 4}(\lambda)$ are invertible for each $\lambda \in \overline{\mathbf{C}^{+}}$. Then, using (2.36), we can conclude that $a_{l 4}(\lambda)$ and $a_{r 1}(\lambda)$ are invertible for each $\lambda \in \overline{\mathbf{C}^{-}}$. The proof of (ii) and (iii) follows from (i) and Proposition 2.5.

\section{The Scattering Matrix}

We can write (2.6) as

$$
\begin{aligned}
& a_{l 1}(\lambda) a_{r 1}(\lambda)+a_{l 2}(\lambda) a_{r 3}(\lambda)=I_{n}=a_{r 1}(\lambda) a_{l 1}(\lambda)+a_{r 2}(\lambda) a_{l 3}(\lambda), \\
& a_{l 1}(\lambda) a_{r 2}(\lambda)+a_{l 2}(\lambda) a_{r 4}(\lambda)=0=a_{r 1}(\lambda) a_{l 2}(\lambda)+a_{r 2}(\lambda) a_{l 4}(\lambda),
\end{aligned}
$$




$$
\begin{gathered}
a_{l 3}(\lambda) a_{r 1}(\lambda)+a_{l 4}(\lambda) a_{r 3}(\lambda)=0=a_{r 3}(\lambda) a_{l 1}(\lambda)+a_{r 4}(\lambda) a_{l 3}(\lambda), \\
a_{l 3}(\lambda) a_{r 2}(\lambda)+a_{l 4}(\lambda) a_{l 4}(\lambda)=I_{n}=a_{r 3}(\lambda) a_{l 2}(\lambda)+a_{r 4}(\lambda) a_{l 4}(\lambda) .
\end{gathered}
$$

Let us define the transmission coefficients $T_{l}(\lambda)$ from the left and $T_{r}(\lambda)$ from the right, and the reflection coefficients $R(\lambda)$ from the right and $L(\lambda)$ from the left, as follows:

$$
\begin{gathered}
T_{l}(\lambda)=a_{l 1}(\lambda)^{-1}, \quad T_{r}(\lambda)=a_{r 4}(\lambda)^{-1}, \\
R(\lambda)=a_{r 2}(\lambda) a_{r 4}(\lambda)^{-1}, \quad L(\lambda)=a_{l 3}(\lambda) a_{l 1}(\lambda)^{-1} .
\end{gathered}
$$

From (3.2), (3.3), and (3.6) we see that

$$
R(\lambda)=-a_{l 1}(\lambda)^{-1} a_{l 2}(\lambda), \quad L(\lambda)=-a_{r 4}(\lambda)^{-1} a_{r 3}(\lambda) .
$$

Note that using (2.3) and (3.1)-(3.7), we can express the matrices in (2.22) in terms of the scattering coefficients as

$$
\begin{gathered}
a_{l}(\lambda)=\left[\begin{array}{cc}
T_{l}(\lambda)^{-1} & -T_{l}(\lambda)^{-1} R(\lambda) \\
L(\lambda) T_{l}(\lambda)^{-1} & {\left[T_{r}(\lambda)^{\dagger}\right]^{-1}}
\end{array}\right], \\
a_{r}(\lambda)=\left[\begin{array}{cc}
{\left[T_{l}(\lambda)^{\dagger}\right]^{-1}} & R(\lambda) T_{r}(\lambda)^{-1} \\
-T_{r}(\lambda)^{-1} L(\lambda) & T_{r}(\lambda)^{-1}
\end{array}\right],
\end{gathered}
$$

where the off-diagonal entries can be expressed in terms of $L(\lambda)$ or $R(\lambda)$ by using

$$
L(\lambda) T_{l}(\lambda)^{-1}=-\left[R(\lambda) T_{r}(\lambda)^{-1}\right]^{\dagger}
$$

which is immediate from (2.33).

Now let us define the scattering matrix $\mathbf{S}(\lambda)$ associated with (1.1) as

$$
\mathbf{S}(\lambda)=\left[\begin{array}{cc}
T_{l}(\lambda) & R(\lambda) \\
L(\lambda) & T_{r}(\lambda)
\end{array}\right] .
$$

TheOREM 3.1. The scattering matrix $\mathbf{S}(\lambda)$ is continuous for $\lambda \in \mathbf{R}$ and converges to $I_{2 n}$ as $\lambda \rightarrow \pm \infty$. It is unitary for each $\lambda \in \mathbf{R}$, and hence the scattering coefficients satisfy

$$
T_{l}(\lambda) T_{l}(\lambda)^{\dagger}+R(\lambda) R(\lambda)^{\dagger}=I_{n}=T_{r}(\lambda)^{\dagger} T_{r}(\lambda)+R(\lambda)^{\dagger} R(\lambda),
$$




$$
\begin{aligned}
& T_{l}(\lambda)^{\dagger} T_{l}(\lambda)+L(\lambda)^{\dagger} L(\lambda)=I_{n}=T_{r}(\lambda) T_{r}(\lambda)^{\dagger}+L(\lambda) L(\lambda)^{\dagger}, \\
& T_{r}(\lambda) R(\lambda)^{\dagger}+L(\lambda) T_{l}(\lambda)^{\dagger}=0=T_{r}(\lambda)^{\dagger} L(\lambda)+R(\lambda)^{\dagger} T_{l}(\lambda) .
\end{aligned}
$$

Moreover, for $\lambda \in \mathbf{R}$ we have

$$
\begin{gathered}
\operatorname{det} T_{l}(\lambda)=\operatorname{det} T_{r}(\lambda) \\
\operatorname{det}\left[\begin{array}{cc}
I_{n} & R(\lambda) \\
R(\lambda)^{\dagger} & I_{n}
\end{array}\right]=\operatorname{det}\left[\begin{array}{cc}
I_{n} & L(\lambda) \\
L(\lambda)^{\dagger} & I_{n}
\end{array}\right]=\left|\operatorname{det} T_{l}(\lambda)\right|^{2} \\
\operatorname{det} \mathbf{S}(\lambda)=\frac{\operatorname{det} T_{l}(\lambda)}{\left[\operatorname{det} T_{l}(\lambda)\right]^{*}}
\end{gathered}
$$

Proof. The continuity and the large- $\lambda$ asymptotics follow from Propositions 2.5 and 2.10. Using (3.8)-(3.10) in (2.6), we get $\mathbf{S}(\lambda) \mathbf{S}(\lambda)^{\dagger}=I_{2 n}$, from which (3.12)-(3.14) follow. From (2.38), (3.8), and (3.9) we obtain (3.15). Using (3.10), we can write (3.8) and (3.9) as

$$
\begin{aligned}
& a_{l}(\lambda)=\left[\begin{array}{cc}
T_{l}(\lambda)^{-1} & 0 \\
0 & {\left[T_{r}(\lambda)^{\dagger}\right]^{-1}}
\end{array}\right]\left[\begin{array}{cc}
I_{n} & -R(\lambda) \\
-R(\lambda)^{\dagger} & I_{n}
\end{array}\right], \\
& a_{r}(\lambda)=\left[\begin{array}{cc}
{\left[T_{l}(\lambda)^{\dagger}\right]^{-1}} & 0 \\
0 & T_{r}(\lambda)^{-1}
\end{array}\right]\left[\begin{array}{cc}
I_{n} & -L(\lambda)^{\dagger} \\
-L(\lambda) & I_{n}
\end{array}\right],
\end{aligned}
$$

and hence, using $(2.1),(3.15),(3.18),(3.19)$, and $\operatorname{det} J_{2 n}=(-1)^{n}$, we get (3.16). Using $(2.2),(2.34),(3.5)$, and (3.6) it follows that

$$
f_{-}(x, \lambda)=f_{+}(x, \lambda) J_{2 n} \mathbf{S}(\lambda) J_{2 n}, \quad \lambda \in \mathbf{R}
$$

Thus, from $(3.5),(2.38),(2.40),(3.20)$, and $\operatorname{det} J_{2 n}=(-1)^{n}$, we obtain $(3.17)$.

In Proposition 2.10 we have seen that $a_{l 1}(\lambda)$ and $a_{r 4}(\lambda)$ have invertible, continuous, and analytic extensions from the real axis to $\mathbf{C}^{+}$. Thus, from (3.5) and Proposition 2.10, we obtain the following result.

COROLlary 3.2. The transmission coefficients $T_{l}(\lambda)$ and $T_{r}(\lambda)$ and their inverses $T_{l}(\lambda)^{-1}$ and $T_{r}(\lambda)^{-1}$ are continuous in $\lambda \in \overline{\mathbf{C}^{+}}$and analytic in $\lambda \in \mathbf{C}^{+} ;$these four matrices all converge to $I_{n}$ as $\lambda \rightarrow \infty$ in $\overline{\mathbf{C}^{+}}$. Similarly, the matrices $T_{l}\left(\lambda^{*}\right)^{\dagger}$ and $T_{r}\left(\lambda^{*}\right)^{\dagger}$ and their 
inverses $\left[T_{l}\left(\lambda^{*}\right)^{\dagger}\right]^{-1}$ and $\left[T_{r}\left(\lambda^{*}\right)^{\dagger}\right]^{-1}$ are continuous in $\lambda \in \overline{\mathbf{C}^{-}}$and analytic in $\lambda \in \mathbf{C}^{-}$; these four matrices all converge to $I_{n}$ as $\lambda \rightarrow \infty$ in $\overline{\mathbf{C}^{-}}$.

In general, $R(\lambda)$ and $L(\lambda)$ do not have analytic continuations off the real axis. In the special case when $k(x)$ vanishes on a half line, we have the following.

Proposition 3.3. If $k(x)$ is supported in the right half line $\mathbf{R}^{+}$, then $L(\lambda)$ extends to a function that is continuous on $\overline{\mathbf{C}^{+}}$, is analytic on $\mathbf{C}^{+}$, and vanishes as $\lambda \rightarrow \infty$ in $\overline{\mathbf{C}^{+}}$. Similarly, if $k(x)$ is supported in the left half line $\mathbf{R}^{-}$, then $R(\lambda)$ extends to a function that is continuous on $\overline{\mathbf{C}^{+}}$, is analytic on $\mathbf{C}^{+}$, and vanishes as $\lambda \rightarrow \infty$ in $\overline{\mathbf{C}^{+}}$.

Proof. If $k$ has support in $\mathbf{R}^{+}$, then from (2.27) and Proposition 2.3 we see that $a_{l 3}(\lambda)$ has an extension that is continuous in $\lambda \in \overline{\mathbf{C}^{+}}$, is analytic in $\lambda \in \mathbf{C}^{+}$, and converges to 0 as $\lambda \rightarrow \infty$ in $\overline{\mathbf{C}^{+}}$. Thus, using (3.6) and Corollary 3.2, we can conclude that $L(\lambda)$ extends to a function that is continuous on $\overline{\mathbf{C}^{+}}$, is analytic on $\mathbf{C}^{+}$, and vanishes as $\lambda \rightarrow \infty$ in $\overline{\mathbf{C}^{+}}$. In a similar manner, if $k$ is supported in the left half line $\mathbf{R}^{-}$, using (2.30), (3.6), Proposition 2.3, and Corollary 3.2, we obtain that $R(\lambda)$ extends to a function that is continuous on $\overline{\mathbf{C}^{+}}$, is analytic on $\mathbf{C}^{+}$, and vanishes as $\lambda \rightarrow \infty$ in $\overline{\mathbf{C}^{+}}$.

Considering $H=-i J_{2 n}(d / d x)-V(x)$ and $H_{0}=-i J_{2 n}(d / d x)$ as the perturbed and free Hamiltonians, respectively, one can prove the existence of the Møller wave operators

$$
W_{ \pm}=\lim _{t \rightarrow \pm \infty} e^{i t H} e^{-i t H_{0}}
$$

as (partial) isometries and construct $[1,37]$ the scattering operator $S=W_{+} W_{-}^{*}$. Using the Fourier transform

$$
(\mathcal{F} \phi)(\lambda)=\int_{-\infty}^{\infty} d \alpha e^{i \lambda J_{2 n} \alpha} \phi(\alpha), \quad \phi \in L^{2}\left(\mathbf{R} ; \mathbf{C}^{2 n \times 1}\right),
$$

one can prove that $\left(\mathcal{F} S \mathcal{F}^{-1} \phi\right)(\lambda)=\mathbf{S}(\lambda) \phi(\lambda)$, where $\mathbf{S}(\lambda)$ is given by (3.11). In other words, $\mathbf{S}(\lambda)$ coincides with the scattering matrix obtained from time-dependent scattering theory. 


\section{Fourier Transforms}

Let $\mathcal{W}^{q}$ denote the Wiener algebra of all $q \times q$ matrix functions of the form

$$
Z(\lambda)=Z_{\infty}+\int_{-\infty}^{\infty} d \alpha z(\alpha) e^{i \lambda \alpha}
$$

where $z(\alpha)$ is a $q \times q$ matrix function whose entries belong to $L^{1}(\mathbf{R})$ and $Z_{\infty}=Z( \pm \infty)$. Then $\mathcal{W}^{q}$ is a Banach algebra with unit element with respect to the norm

$$
\|Z\|_{\mathcal{W}^{q}}=\left\|Z_{\infty}\right\|+\int_{-\infty}^{\infty} d \alpha\|z(\alpha)\|
$$

and its invertible elements are those $Z(\lambda)$ as in (4.1) for which $Z_{\infty}$ and $Z(\lambda)$ for all $\lambda \in \mathbf{R}$ are nonsingular matrices [23]. We will use $\mathcal{W}_{ \pm}^{q}$ to denote the subalgebra of those functions $Z(\lambda)$ for which $z(\alpha)$ has support in $\mathbf{R}^{ \pm}$and $\mathcal{W}_{ \pm, 0}^{q}$ to denote the subalgebra of those functions $Z(\lambda)$ for which $Z_{\infty}=0$ and $z(\alpha)$ has support in $\mathbf{R}^{ \pm}$. Then, $\mathcal{W}^{q}=\mathcal{W}_{+}^{q} \oplus \mathcal{W}_{-, 0}^{q}=$ $\mathcal{W}_{+, 0}^{q} \oplus \mathcal{W}_{-}^{q}$

In this section we prove that the matrix functions $M_{l}(x, \cdot), M_{r}(x, \cdot)$, and $\mathbf{S}(\cdot)$ belong to $\mathcal{W}^{2 n}$, and that $m_{ \pm}(x, \cdot)$ belongs to $\mathcal{W}_{ \pm}^{2 n}$. Contrary to the case of the usual Schrödinger equation with potentials in $L_{1}^{1}(\mathbf{R})$, we cannot exploit estimates $[20,21]$ of the type $M_{l}(x, \lambda)=I_{2 n}+O(1 / \lambda)$ as $\lambda \rightarrow \pm \infty$ to construct the function $z(\alpha)$ in (4.1) first as an $L^{2}$-matrix function and then go on to prove that it in fact belongs to $L^{1}(\mathbf{R})$.

Let us construct the $L^{1}$-matrix functions $b_{ \pm}(x, \cdot), B_{l}(x, \cdot)$, and $B_{r}(x, \cdot)$ such that

$$
\begin{gathered}
m_{ \pm}(x, \lambda)=I_{2 n}+\int_{0}^{\infty} d \alpha b_{ \pm}(x, \alpha) e^{ \pm i \lambda \alpha}, \\
M_{l}(x, \lambda)=I_{2 n}+\int_{0}^{\infty} d \alpha B_{l}(x, \alpha) e^{i \lambda J_{2 n} \alpha}, \quad M_{r}(x, \lambda)=I_{2 n}+\int_{0}^{\infty} d \alpha B_{r}(x, \alpha) e^{-i \lambda J_{2_{n} \alpha}} .
\end{gathered}
$$

Indeed, partitioning the matrix functions $B_{l}(x, \alpha)$ and $B_{r}(x, \alpha)$ in (4.3) into $n \times n$ blocks as

$$
B_{l}(x, \alpha)=\left[\begin{array}{ll}
B_{l 1}(x, \alpha) & B_{l 2}(x, \alpha) \\
B_{l 3}(x, \alpha) & B_{l 4}(x, \alpha)
\end{array}\right], \quad B_{r}(x, \alpha)=\left[\begin{array}{ll}
B_{r 1}(x, \alpha) & B_{r 2}(x, \alpha) \\
B_{r 3}(x, \alpha) & B_{r 4}(x, \alpha)
\end{array}\right]
$$


so that

$$
b_{+}(x, \alpha)=\left[\begin{array}{ll}
B_{l 1}(x, \alpha) & B_{r 2}(x, \alpha) \\
B_{l 3}(x, \alpha) & B_{r 4}(x, \alpha)
\end{array}\right], \quad b_{-}(x, \alpha)=\left[\begin{array}{cc}
B_{r 1}(x, \alpha) & B_{l 2}(x, \alpha) \\
B_{r 3}(x, \alpha) & B_{l 4}(x, \alpha)
\end{array}\right]
$$

we apply (4.3) to (2.12) and (2.17), and derive the coupled integral equations for $\alpha>0$

$$
\begin{gathered}
B_{l 1}(x, \alpha)=-i \int_{x}^{\infty} d y k(y) B_{l 3}(y, \alpha), \\
B_{l 2}(x, \alpha)=-\frac{i}{2} k(x+\alpha / 2)-i \int_{x}^{x+\alpha / 2} d y k(y) B_{l 4}(y, \alpha+2 x-2 y), \\
B_{l 3}(x, \alpha)=\frac{i}{2} k(x+\alpha / 2)^{\dagger}+i \int_{x}^{x+\alpha / 2} d y k(y)^{\dagger} B_{l 1}(y, \alpha+2 x-2 y), \\
B_{l 4}(x, \alpha)=i \int_{x}^{\infty} d y k(y)^{\dagger} B_{l 2}(y, \alpha), \\
B_{r 1}(x, \alpha)=i \int_{-\infty}^{x} d y k(y) B_{r 3}(y, \alpha), \\
B_{r 2}(x, \alpha)=\frac{i}{2} k(x-\alpha / 2)+i \int_{x-\alpha / 2}^{x} d y k(y) B_{r 4}(y, \alpha+2 y-2 x), \\
B_{r 3}(x, \alpha)=-\frac{i}{2} k(x-\alpha / 2)^{\dagger}-i \int_{x-\alpha / 2}^{x} d y k(y)^{\dagger} B_{l 1}(y, \alpha+2 y-2 x), \\
B_{r 4}(x, \alpha)=-i \int_{-\infty}^{x} d y k(y)^{\dagger} B_{r 2}(y, \alpha) .
\end{gathered}
$$

We first prove that, for each $x \in \mathbf{R}$, the four systems of integral equations (4.5) and (4.7), (4.6) and (4.8), (4.9) and (4.11), (4.10) and (4.12) have unique solutions with entries in $L^{1}\left(\mathbf{R}^{+}\right)$. Then for the matrix functions $m_{ \pm}(x, \lambda), M_{l}(x, \lambda)$, and $M_{r}(x, \lambda)$ defined in (4.2) and (4.3), we derive the integral relations (2.13)-(2.16) and (2.18)-(2.21). In this way we will have proved that $M_{l}(x, \cdot)$ and $M_{r}(x, \cdot)$ belong to $\mathcal{W}^{2 n}$ and $m_{ \pm}(x, \cdot)$ belongs to $\mathcal{W}_{ \pm}^{2 n}$.

Let us introduce the following mixed norm on the $2 n \times 2 n$ matrix functions $B(x, \alpha)$ depending on $(x, \alpha) \in \mathbf{R} \times \mathbf{R}^{+}$:

$$
\|B(\cdot, \cdot)\|_{\infty, 1}=\sup _{x \in \mathbf{R}}\|B(x, \cdot)\|_{1} \cdot
$$


TheOREM 4.1. Assume that the entries of $k(x)$ belong to $L^{1}(\mathbf{R})$. Then, for each $x \in \mathbf{R}$, the four systems of integral equations (4.5) and (4.7), (4.6) and (4.8), (4.9) and (4.11), (4.10) and (4.12) have unique solutions with finite mixed norm as defined in (4.13). Consequently, $m_{+}(x, \cdot)$ belongs to $\mathcal{W}_{+}^{2 n}, m_{-}(x, \cdot)$ belongs to $\mathcal{W}_{-}^{2 n}$, and $M_{l}(x, \cdot)$ and $M_{r}(x, \cdot)$ belong to $\mathcal{W}^{2 n}$.

Proof. Consider (4.5) and (4.7). We can solve this system by iteration as follows. Define

$$
\begin{gathered}
B_{l 3}^{(0)}(x, \alpha)=0, \\
B_{l 1}^{(j)}(x, \alpha)=-i \int_{x}^{\infty} d y k(y) B_{l 3}^{(j)}(y, \alpha), \quad j \geq 0, \\
B_{l 3}^{(j+1)}(x, \alpha)=\frac{i}{2} k(x+\alpha / 2)^{\dagger}+i \int_{x}^{x+\alpha / 2} d y k(y)^{\dagger} B_{l 1}^{(j)}(y, \alpha+2 x-2 y), \quad j \geq 0 .
\end{gathered}
$$

Taking operator norms we obtain from (4.14) and (4.15)

$$
\begin{gathered}
\left\|B_{l 1}^{(j)}(x, \alpha)\right\| \leq \int_{x}^{\infty} d y\|k(y)\|\left\|B_{l 3}^{(j)}(y, \alpha)\right\|, \\
\left\|B_{l 3}^{(j+1)}(x, \alpha)\right\| \leq \frac{1}{2}\|k(x+\alpha / 2)\|+\int_{x}^{x+\alpha / 2} d y\|k(y)\|\left\|B_{l 1}^{(j)}(y, \alpha+2 x-2 y)\right\| .
\end{gathered}
$$

Then the norms of $B_{l 1}^{(j)}(x, \cdot)$ and $B_{l 3}^{(j)}(x, \cdot)$ in $L^{1}\left(\mathbf{R}^{+}\right)$satisfy

$$
\begin{gathered}
\left\|B_{l 1}^{(j)}(x, \cdot)\right\|_{1} \leq \int_{x}^{\infty} d y\|k(y)\|\left\|B_{l 3}^{(j)}(y, \cdot)\right\|_{1}, \\
\left\|B_{l 3}^{(j+1)}(x, \cdot)\right\|_{1} \leq \sigma_{+}(x)+\int_{0}^{\infty} d \alpha \int_{x}^{x+\alpha / 2} d y\|k(y)\|\left\|B_{l 1}^{(j)}(y, \alpha+2 x-2 y)\right\| \\
=\sigma_{+}(x)+\int_{x}^{\infty} d y\|k(y)\|\left\|B_{l 1}^{(j)}(y, \cdot)\right\|_{1},
\end{gathered}
$$

where $\sigma_{+}(x)$ is the quantity defined in (2.11), the order of integration has been changed, and the change of variable $\gamma=\alpha+2 x-2 y$ has been applied. From (4.16) and (4.17) we obtain by induction

$$
\left\|B_{l 1}^{(j)}(x, \cdot)\right\|_{1} \leq \sum_{s=1}^{j} \frac{\sigma_{+}(x)^{2 s}}{(2 s) !}, \quad\left\|B_{l 3}^{(j)}(x, \cdot)\right\|_{1} \leq \sum_{s=1}^{j} \frac{\sigma_{+}(x)^{2 s-1}}{(2 s-1) !}, \quad j \geq 1 .
$$


Consequently,

$$
\left\|B_{l 1}(x, \cdot)\right\|_{1}+\left\|B_{l 3}(x, \cdot)\right\|_{1} \leq \sum_{s=1}^{\infty} \frac{\sigma_{+}(x)^{s}}{s !}=\exp \left(\sigma_{+}(x)\right)-1 .
$$

At the same time we have proved that, for each $x \in \mathbf{R}$, the system of equations (4.5) and (4.7) has a unique solution with entries belonging to $L^{1}\left(\mathbf{R}^{+}\right)$.

The proofs for the three other systems of equations, namely (4.6) and (4.8), (4.9) and (4.11), (4.10) and (4.12) are analogous. In each case we get the estimates

$$
\left\|B_{l j}(x, \cdot)\right\|_{1} \leq \exp \left(\sigma_{+}(x)\right)-1, \quad\left\|B_{r j}(x, \cdot)\right\|_{1} \leq \exp \left(\sigma_{-}(x)\right)-1
$$

where $j=1,2,3,4$.

The integral equations (4.5)-(4.12) allow one to derive the following relations for the potential $k(x)$;

$$
k(x)=2 i B_{l 2}\left(x, 0^{+}\right)=-2 i B_{r 2}\left(x, 0^{+}\right)=2 i B_{l 3}\left(x, 0^{+}\right)^{\dagger}=-2 i B_{r 3}\left(x, 0^{+}\right)^{\dagger} .
$$

To justify (4.19), let us fix $\alpha>0$ and integrate the norm of the left-hand side in (4.7) with respect to $x \in \mathbf{R}$. We obtain

$$
\begin{aligned}
\left\|B_{l 3}(\cdot, \alpha)\right\|_{1} & \leq \frac{1}{2}\|k\|_{1}+\int_{-\infty}^{\infty} d s \int_{y-\alpha / 2}^{y} d x\|k(y)\|\left\|B_{l 1}(y, \alpha+2 x-2 y)\right\| \\
& =\frac{1}{2}\left[\|k\|_{1}+\int_{-\infty}^{\infty} d y \int_{0}^{\alpha} d z\|k(y)\|\left\|B_{l 1}(y, z)\right\|\right] \\
& \leq \frac{1}{2}\left[\|k\|_{1}+\int_{-\infty}^{\infty} d y\|k(y)\|\left\|B_{l 1}(y, \cdot)\right\|_{1}\right] \leq \frac{1}{2}\left[\exp \left(\|k\|_{1}\right)-1\right],
\end{aligned}
$$

where we have used (4.18). Hence, for each $\alpha>0, B_{l 3}(\cdot, \alpha)$ is a matrix function with entries in $L^{1}(\mathbf{R})$. We now easily derive the estimate

$$
\left\|B_{l 3}(\cdot, \alpha)-\frac{i}{2} k(\cdot+\alpha / 2)^{\dagger}\right\|_{1} \leq \frac{1}{2} \int_{-\infty}^{\infty} d y\|k(y)\| \int_{0}^{\alpha} d z\left\|B_{l 1}(y, z)\right\|=o(1), \quad \alpha \rightarrow 0^{+},
$$

which justifies the identity $k(x)=2 i B_{l 3}\left(x, 0^{+}\right)^{\dagger}$. In an analogous way one proves the similar result for $B_{l 2}(\cdot, \alpha), B_{r 2}(\cdot, \alpha)$, and $B_{r 3}(\cdot, \alpha)$. 
THEOREM 4.2. The reflection coefficients $R(\lambda)$ and $L(\lambda)$ belong to $\mathcal{W}^{n}$, and $R( \pm \infty)=$ $L( \pm \infty)=0$. The transmission coefficients $T_{l}(\lambda)$ and $T_{r}(\lambda)$ belong to $\mathcal{W}_{+}^{n}$, and they converge to $I_{n}$ as $\lambda \rightarrow \infty$ in $\overline{\mathbf{C}^{+}}$.

Proof. Using (2.3) and (2.8) we get

$$
\begin{aligned}
& a_{l}(\lambda)=e^{-i \lambda J_{2 n} x} J_{2 n} M_{r}(x, \lambda)^{\dagger} J_{2 n} M_{l}(x, \lambda) e^{i \lambda J_{2 n} x} \\
& a_{r}(\lambda)=e^{-i \lambda J_{2 n} x} J_{2 n} M_{l}(x, \lambda)^{\dagger} J_{2 n} M_{r}(x, \lambda) e^{i \lambda J_{2 n} x}
\end{aligned}
$$

From Theorem 4.1 we see that $M_{l}(x, \lambda)$ and $M_{r}(x, \lambda)$ belong to $\mathcal{W}^{2 n}$. Using $(4.20)$ and (4.21) for $x=0$, we can show that $a_{l}(\lambda)$ and $a_{r}(\lambda)$ are products of elements of $\mathcal{W}^{2 n}$ and hence belong to $\mathcal{W}^{2 n}$. Using (3.5)-(3.7) and Proposition 2.10, we complete the proof of the theorem.

\section{WiENER-Hopf FACTORIZATION}

Using (2.34), (2.41), (2.42), and (3.20), we obtain

$$
m_{-}(x, \lambda)=m_{+}(x, \lambda) \mathbf{G}(x, \lambda), \quad \lambda \in \mathbf{R},
$$

where $\mathbf{G}(x, \lambda)$ is the dilated scattering matrix given by

$$
\mathbf{G}(x, \lambda)=e^{i \lambda J_{2 n} x} J_{2 n} \mathbf{S}(\lambda) J_{2 n} e^{-i \lambda J_{2 n} x}=\left[\begin{array}{cc}
T_{l}(\lambda) & -R(\lambda) e^{2 i \lambda x} \\
-L(\lambda) e^{-2 i \lambda x} & T_{r}(\lambda)
\end{array}\right] .
$$

Since $\mathbf{S}(\lambda)$ is unitary, so is $\mathbf{G}(x, \lambda)$. Hence, we can write (5.1) also in the form

$$
m_{+}(x, \lambda)=m_{-}(x, \lambda) \mathbf{G}(x, \lambda)^{\dagger}, \quad \lambda \in \mathbf{R} .
$$

We have the following result.

THEOREM 5.1. Assume the entries of $k(x)$ belong to $L^{1}(\mathbf{R})$. Then, for each $x \in \mathbf{R}$, $\mathbf{G}(x, \cdot)$ has a unique left-canonical Wiener-Hopf factorization

$$
\mathbf{G}(x, \lambda)=m_{+}(x, \lambda)^{-1} m_{-}(x, \lambda), \quad \lambda \in \mathbf{R},
$$


where $m_{+}(x, \cdot)$ and $m_{+}(x, \cdot)^{-1}$ belong to $\mathcal{W}_{+}^{2 n}, m_{-}(x, \cdot)$ and $m_{-}(x, \cdot)^{-1}$ belong to $\mathcal{W}_{-}^{2 n}$, $m_{+}(x, \lambda)$ and $m_{+}(x, \lambda)^{-1}$ tend to $I_{2 n}$ as $\lambda \rightarrow \infty$ in $\overline{\mathbf{C}^{+}}$, and $m_{-}(x, \lambda)$ and $m_{-}(x, \lambda)^{-1}$ tend to $I_{2 n}$ as $\lambda \rightarrow \infty$ in $\overline{\mathbf{C}^{-}}$.

Proof. Using (3.5) and (3.7) we write

$$
\mathbf{G}(x, \lambda)=\left[\begin{array}{cc}
T_{l}(\lambda) & 0 \\
0 & T_{r}(\lambda)
\end{array}\right]\left[\begin{array}{cc}
I_{n} & a_{l 2}(\lambda) e^{2 i \lambda x} \\
a_{r 3}(\lambda) e^{-2 i \lambda x} & I_{n}
\end{array}\right],
$$

where the right factor has a positive selfadjoint real part when $\lambda \in \mathbf{R}$, and, as seen in Theorem 4.2, the left factor and its inverse belong to $\mathcal{W}_{+}^{2 n}$ and tend to $I_{2 n}$ as $\lambda \rightarrow \infty$ in $\overline{\mathbf{C}^{+}}$. Thus the right factor has a unique left-canonical Wiener-Hopf factorization [19] of the form

$$
\left[\begin{array}{cc}
I_{n} & a_{l 2}(\lambda) e^{2 i \lambda x} \\
a_{r 3}(\lambda) e^{-2 i \lambda x} & I_{n}
\end{array}\right]=\mathbf{W}_{+}(\lambda) \mathbf{W}_{-}(\lambda), \quad \lambda \in \mathbf{R},
$$

where $\mathbf{W}_{ \pm}(\lambda)$ and $\mathbf{W}_{ \pm}(\lambda)^{-1}$ are continuous in $\lambda \in \overline{\mathbf{C}^{ \pm}}$, are analytic in $\lambda \in \mathbf{C}^{ \pm}$, and tend to $I_{2 n}$ as $\lambda \rightarrow \infty$ in $\overline{\mathbf{C}^{ \pm}}$. From (2.38) and (2.40)-(2.42), we have

$$
\begin{array}{cl}
\operatorname{det} m_{+}(x, \lambda)=\frac{1}{\operatorname{det} T_{l}(\lambda)}, & \lambda \in \overline{\mathbf{C}^{+}}, \\
\operatorname{det} m_{-}(x, \lambda)=\frac{1}{\operatorname{det} T_{l}\left(\lambda^{*}\right)^{\dagger}}, & \lambda \in \overline{\mathbf{C}^{-}} .
\end{array}
$$

Hence with the help of Theorems 4.1 and 4.2 , we conclude that $m_{ \pm}(x, \lambda)$ and $m_{ \pm}(x, \lambda)^{-1}$ belong to $\mathcal{W}_{ \pm}^{2 n}$ and tend to $I_{2 n}$ as $\lambda \rightarrow \infty$ in $\overline{\mathbf{C}^{ \pm}}$. Thus, with

$$
m_{+}(x, \lambda)=\mathbf{W}_{+}(\lambda)^{-1}\left(T_{l}(\lambda)^{-1} \oplus T_{r}(\lambda)^{-1}\right), \quad m_{-}(x, \lambda)=\mathbf{W}_{-}(\lambda)
$$

we get the left-canonical factorization in (5.4).

The matrix $V(x)$ can be recovered from the scattering matrix $\mathbf{S}(\lambda)$ as follows. First, form the matrix function $\mathbf{G}(x, \lambda)$ as in (5.2). Next, obtain the Wiener-Hopf factors of $\mathbf{G}(x, \lambda)$ as in (5.4). One can then recover $V(x)$ as

$$
V(x)=-i J_{2 n} m_{ \pm}^{\prime}(x, 0) m_{ \pm}(x, 0)^{-1}
$$


Indeed, from (1.1) using (2.41) and (2.42) we easily derive

$$
-i J_{2 n} m_{ \pm}^{\prime}(x, \lambda)-V(x) m_{ \pm}(x, \lambda)=\lambda\left[m_{ \pm}(x, \lambda)-J_{2 n} m_{ \pm}(x, \lambda) J_{2 n}\right]
$$

which implies (5.5). To make (5.5) a viable way of computing the potential $k(x)$ from the Wiener-Hopf factors of $(5.2)$, one needs to prove that $m_{ \pm}(x, 0)$ are absolutely continuous and that the entries of the $2 n \times 2 n$ matrix on the right-hand side in (5.5) belong to $L^{1}(\mathbf{R})$.

If the potential $k$ is outside the class $L^{1}(\mathbf{R})$, the matrices $a_{l}(\lambda)$ and $a_{r}(\lambda)$ may fail to be continuous at $\lambda=0$; in this case the scattering matrix $\mathbf{S}(\lambda)$ might not have a canonical Wiener-Hopf factorization and the inverse problem may have a one-parameter family of nonintegrable potentials as its solutions. For example, let $n=1$ and use the scattering coefficients

$$
R(\lambda)=L(\lambda)=-\frac{i}{\lambda+i}, \quad T_{l}(\lambda)=T_{r}(\lambda)=\frac{\lambda}{\lambda+i}
$$

Note that (3.12)-(3.14) are satisfied, but $T_{l}(0)=0$ so that $T_{l}(\lambda)^{-1}$ is not continuous at $\lambda=0$. Hence, in view of Corollary 3.2, the scattering coefficients in (5.6) do not correspond to an $L^{1}$-potential. In fact, there is a one-parameter family of potentials $k(x, \xi)$ given by

$$
k(x, \xi)=\theta(-x) \frac{2 i}{1+2 \xi-2 x}+\theta(x) \frac{-4 i \xi}{1+2 \xi+4 \xi x}
$$

where $\theta(x)$ is the Heaviside function and $\xi \in[0,+\infty)$ is the parameter. The matrices $m_{ \pm}(x, \lambda)$ defined in (2.41) and (2.42) are obtained as

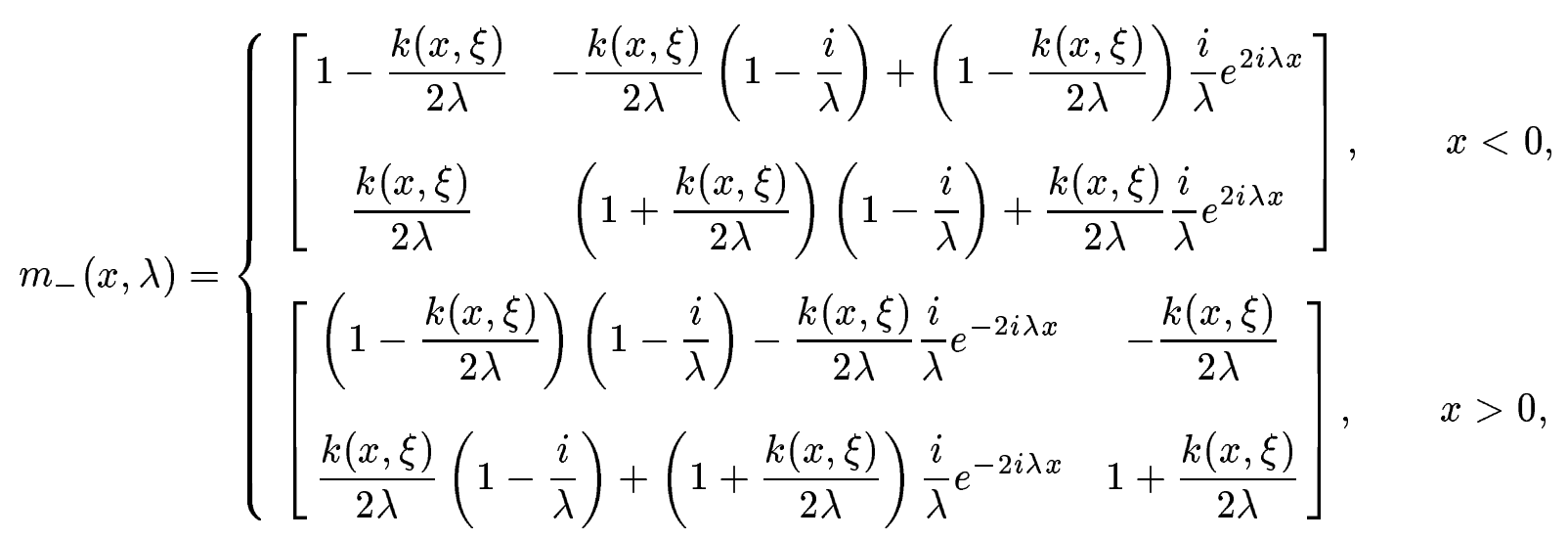




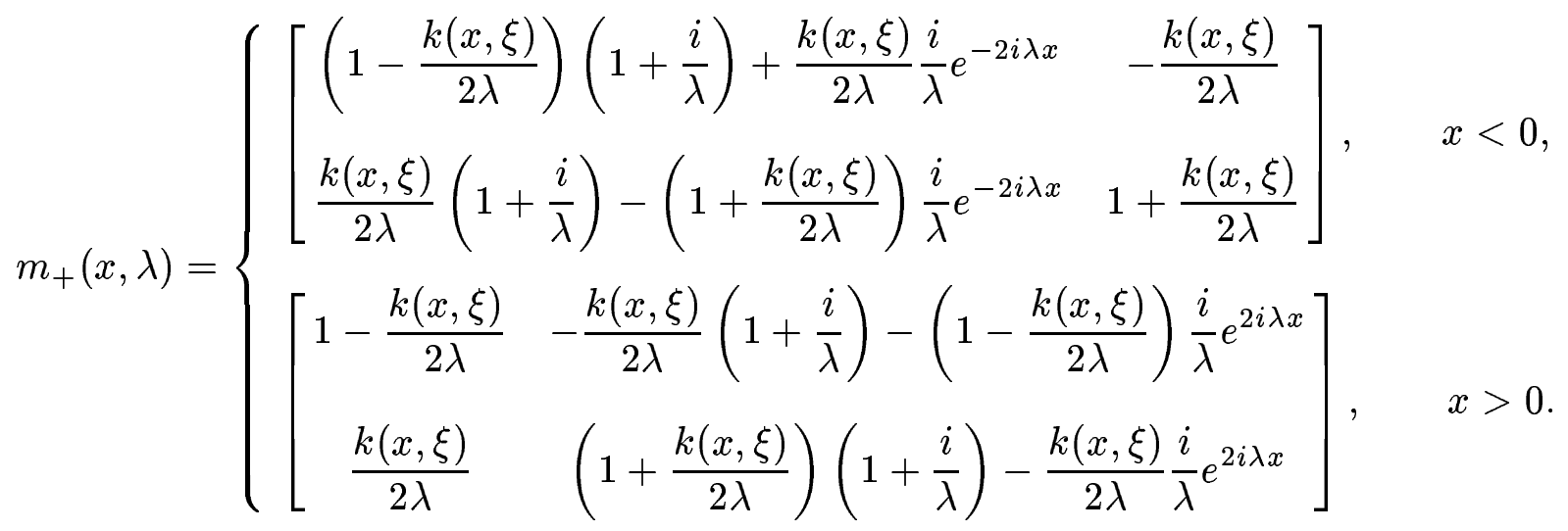

One easily finds that

$$
a_{l}(\lambda)=a_{r}(\lambda)^{-1}=\left[\begin{array}{cc}
1+\frac{i}{\lambda} & \frac{i}{\lambda} \\
-\frac{i}{\lambda} & 1-\frac{i}{\lambda}
\end{array}\right]
$$

is discontinuous at $\lambda=0$. Moreover, the left Wiener-Hopf factorization for $\mathbf{G}(x, \lambda)$ corresponding to (5.6) is noncanonical and given by

$$
\mathbf{G}(x, \lambda)=\left[\begin{array}{cc}
\frac{\lambda}{\lambda+i} & \frac{i}{\lambda+i} e^{2 i \lambda x} \\
\frac{i}{\lambda+i} e^{-2 i \lambda x} & \frac{\lambda}{\lambda+i}
\end{array}\right]=\mathbf{G}_{+}(x, \lambda) \mathbf{D}(\lambda) \mathbf{G}_{-}(x, \lambda)
$$

where

$$
\begin{gathered}
\mathbf{G}_{+}(x, \lambda)=m_{+}(x, \lambda)^{-1}\left[\left(\frac{\lambda+i}{\lambda}\right) \mathbf{Q}_{-}+\mathbf{Q}_{+}\right] \\
\mathbf{G}_{-}(x, \lambda)=\left[\left(\frac{\lambda}{\lambda-i}\right) \mathbf{Q}_{-}+\mathbf{Q}_{+}\right] m_{-}(x, \lambda) \\
\mathbf{D}(\lambda)=\left(\frac{\lambda-i}{\lambda+i}\right) \mathbf{Q}_{-}+\mathbf{Q}_{+}, \quad \mathbf{Q}_{ \pm}=\frac{1}{2}\left[\begin{array}{cc}
1 & \pm 1 \\
\pm 1 & 1
\end{array}\right]
\end{gathered}
$$

6. The Marchenko Method

Using (4.2) and (5.1) we get

$$
m_{+}(x, \lambda)\left[\mathbf{G}(x, \lambda)-I_{2 n}\right]=\int_{-\infty}^{\infty} d \alpha\left[b_{-}(x, \alpha)-b_{+}(x,-\alpha)\right] e^{-i \lambda \alpha}, \quad \lambda \in \mathbf{R} .
$$

From (5.2) and Theorem 4.2 we conclude that

$$
\mathbf{G}(x, \lambda)-I_{2 n}=\int_{-\infty}^{\infty} d \alpha \mathbf{H}(\alpha) e^{i \lambda \alpha}, \quad \lambda \in \mathbf{R}
$$


with

$$
\mathbf{H}(\alpha)=\left[\begin{array}{cc}
\nu_{l}(\alpha) & -\hat{R}(2 x-\alpha) \\
-\hat{L}(-2 x-\alpha) & \nu_{r}(\alpha)
\end{array}\right], \quad \alpha \in \mathbf{R},
$$

where the hat is used to denote the Fourier transform so that

$$
\begin{array}{cc}
R(\lambda)=\int_{-\infty}^{\infty} d \alpha \hat{R}(\alpha) e^{-i \lambda \alpha}, & L(\lambda)=\int_{-\infty}^{\infty} d \alpha \hat{L}(\alpha) e^{-i \lambda \alpha}, \\
T_{l}(\lambda)=I_{n}+\int_{-\infty}^{\infty} d \alpha \nu_{l}(\alpha) e^{i \lambda \alpha}, & T_{r}(\lambda)=I_{n}+\int_{-\infty}^{\infty} d \alpha \nu_{r}(\alpha) e^{i \lambda \alpha} .
\end{array}
$$

By Theorem 4.2, $\nu_{l}(\alpha)$ and $\nu_{r}(\alpha)$ vanish for $\alpha>0$ and their entries belong to $L^{1}\left(\mathbf{R}^{+}\right)$, and the entries of $\hat{R}(\cdot)$ and $\hat{L}(\cdot)$ belong to $L^{1}(\mathbf{R})$. Let us define

$$
g(x, \alpha)=\left[\begin{array}{cc}
0 & -\hat{R}(2 x+\alpha) \\
-\hat{L}(-2 x+\alpha) & 0
\end{array}\right], \quad \alpha>0 .
$$

Upon writing

$$
m_{+}(x, \lambda)\left[\mathbf{G}(x, \lambda)-I_{2 n}\right]=\left[\mathbf{G}(x, \lambda)-I_{2 n}\right]+\left[m_{+}(x, \lambda)-I_{2 n}\right]\left[\mathbf{G}(x, \lambda)-I_{2 n}\right],
$$

by using (6.1) on the left-hand side, (4.2) and (6.2)-(6.6) on the right-hand side, together with the convolution theorem and the fact that $b_{+}(x, \cdot)$ and $b_{-}(x, \cdot)$ have their supports in $\mathbf{R}^{+}$, we obtain the $2 n \times 2 n$ system of coupled Marchenko equations

$$
b_{-}(x, \alpha)=g(x, \alpha)+\int_{0}^{\infty} d \beta b_{+}(x, \beta) g(x, \alpha+\beta), \quad \alpha>0 .
$$

Similarly, using

$$
m_{-}(x, \lambda)\left[\mathbf{G}(x, \lambda)^{\dagger}-I_{2 n}\right]=\left[\mathbf{G}(x, \lambda)^{\dagger}-I_{2 n}\right]+\left[m_{-}(x, \lambda)-I_{2 n}\right]\left[\mathbf{G}(x, \lambda)^{\dagger}-I_{2 n}\right],
$$

we obtain the $2 n \times 2 n$ Marchenko system

$$
b_{+}(x, \alpha)=g(x, \alpha)^{\dagger}+\int_{0}^{\infty} d \beta b_{-}(x, \beta) g(x, \alpha+\beta)^{\dagger}, \quad \alpha>0 .
$$

In fact, using (6.7) in (6.8) and vice versa, we can uncouple them and write the resulting uncoupled systems in an $n \times n$ form with the notations used in (4.4). This leads to the uncoupled Marchenko equations for $\alpha>0$ given by

$$
B_{l 2}(x, \alpha)=-\hat{R}(\alpha+2 x)+\int_{0}^{\infty} d \beta \int_{0}^{\infty} d \gamma B_{l 2}(x, \gamma) \hat{R}(\beta+\gamma+2 x)^{\dagger} \hat{R}(\alpha+\beta+2 x),
$$




$$
\begin{aligned}
& B_{l 3}(x, \alpha)=-\hat{R}(\alpha+2 x)^{\dagger}+\int_{0}^{\infty} d \beta \int_{0}^{\infty} d \gamma B_{l 3}(x, \gamma) \hat{R}(\beta+\gamma+2 x) \hat{R}(\alpha+\beta+2 x)^{\dagger} \\
& B_{r 2}(x, \alpha)=-\hat{L}(\alpha-2 x)^{\dagger}+\int_{0}^{\infty} d \beta \int_{0}^{\infty} d \gamma B_{r 2}(x, \gamma) \hat{L}(\beta+\gamma-2 x) \hat{L}(\alpha+\beta-2 x)^{\dagger}, \\
& B_{r 3}(x, \alpha)=-\hat{L}(\alpha-2 x)+\int_{0}^{\infty} d \beta \int_{0}^{\infty} d \gamma B_{r 3}(x, \gamma) \hat{L}(\beta+\gamma-2 x)^{\dagger} \hat{L}(\alpha+\beta-2 x), \\
& B_{l 1}(x, \alpha)=\int_{0}^{\infty} d \beta \hat{R}(\beta+2 x) \hat{R}(\alpha+\beta+2 x)^{\dagger} \\
&+\int_{0}^{\infty} d \beta \int_{0}^{\infty} d \gamma B_{l 1}(x, \gamma) \hat{R}(\beta+\gamma+2 x) \hat{R}(\alpha+\beta+2 x)^{\dagger}, \\
& B_{l 4}(x, \alpha)= \int_{0}^{\infty} d \beta \hat{R}(\beta+2 x)^{\dagger} \hat{R}(\alpha+\beta+2 x) \\
&+\int_{0}^{\infty} d \beta \int_{0}^{\infty} d \gamma B_{l 4}(x, \gamma) \hat{R}(\beta+\gamma+2 x)^{\dagger} \hat{R}(\alpha+\beta+2 x), \\
&+\int_{0}^{\infty} d \beta \int_{0}^{\infty} d \gamma B_{r 4}(x, \gamma) \hat{L}(\beta+\gamma-2 x) \hat{L}(\alpha+\beta-2 x)^{\dagger} . \\
&+\int_{0}^{\infty} d \beta \int_{0}^{\infty} d \gamma B_{r 1}(x, \gamma) \hat{L}(\beta+\gamma-2 x)^{\dagger} \hat{L}(\alpha+\beta-2 x), \\
& B_{r 1}(x, \alpha)= \int_{0}^{\infty} d \beta \hat{L}(\beta-2 x) \hat{L}(\alpha+\beta-2 x)^{\dagger} \hat{L}(\alpha+\beta-2 x) \\
& B_{r 4}(x, \alpha)=
\end{aligned}
$$

By $L^{1}\left(\mathbf{R}^{+} ; \mathbf{C}^{q \times q}\right)$ we denote the Banach space of all complex $q \times q$ matrix functions $z(\alpha)$ where each entry belongs to $L^{1}\left(\mathbf{R}^{+}\right)$, endowed with the norm $\int_{0}^{\infty} d \alpha\|z(\alpha)\|$.

THEOREM 6.1. The coupled system of Marchenko integral equations (6.7) and (6.8) is uniquely solvable in $L^{1}\left(\mathbf{R}^{+} ; \mathbf{C}^{2 n \times 2 n}\right)$. The integral operator in each of the eight uncoupled Marchenko equations (6.9)-(6.16) is selfadjoint, and each of these eight equations is uniquely solvable in $L^{1}\left(\mathbf{R}^{+} ; \mathbf{C}^{n \times n}\right)$.

Proof. The selfadjointness of the integral operators in (6.9)-(6.16) is clear. From (3.12), (3.13), and Corollary 3.2 it follows that

$$
\sup _{\lambda \in \mathbf{R}}\|L(\lambda)\|<1, \quad \sup _{\lambda \in \mathbf{R}}\|R(\lambda)\|<1
$$


Now observe that the integral operators on $L^{2}\left(\mathbf{R}^{+} ; \mathbf{C}^{n}\right)$ with kernels $(\alpha, \beta) \mapsto \hat{R}(\alpha+\beta+2 x)$, $(\alpha, \beta) \mapsto \hat{R}(\alpha+\beta+2 x)^{\dagger},(\alpha, \beta) \mapsto \hat{L}(\alpha+\beta-2 x)$, and $(\alpha, \beta) \mapsto \hat{L}(\alpha+\beta-2 x)^{\dagger}$ all have the following action: one imbeds $L^{2}\left(\mathbf{R}^{+} ; \mathbf{C}^{n}\right)$ into $L^{2}\left(\mathbf{R} ; \mathbf{C}^{n}\right)$ isometrically, applies the sign flip $h(\alpha) \mapsto h(-\alpha)$ followed by the convolution with an $L^{1}$-matrix function, and then projects orthogonally back onto $L^{2}\left(\mathbf{R}^{+} ; \mathbf{C}^{n}\right)$. Since the Fourier transforms of these matrix functions have norm strictly less than one, this is also the case for the norms of these integral operators. Hence, the system of equations (6.7) and (6.8) as well as each of the eight equations (6.9)-(6.16) are uniquely solvable on the direct sum of a suitable number of copies of $L^{2}\left(\mathbf{R}^{+}\right)$. Since the integral operators are compact as a result of the integrability of $\hat{L}(\cdot)$ and $\hat{R}(\cdot)$, both on $L^{2}$ and on $L^{1}$ (cf. Lemma XII 2.4 of [23], the proof for the $L^{2}$-case there can easily be adapted to cover the $L^{1}$-case; also p. 401 of [18]), the system of equations (6.7) and (6.8) as well as each of the eight equations (6.9)-(6.16) are uniquely solvable on the direct sum of a suitable number of copies of $L^{1}\left(\mathbf{R}^{+}\right)$.

From (4.19), we see that we can recover the potential $k(x)$ by solving either one of the four Marchenko equations (6.9)-(6.12).

The unique solvability of the Marchenko equations (6.9)-(6.16) has a number of other consequences. For example, if $R(\lambda)$ is analytic on $\mathbf{C}^{+}$, then $\hat{R}(\alpha)$ is supported on $\mathbf{R}^{-}$ and hence the right-hand sides in (6.9), (6.10), (6.13), and (6.14) vanish when $x>0$. Since these equations are uniquely solvable, their solutions vanish as well and therefore $k$ is supported on $\mathbf{R}^{-}$. On the other hand, if $L(\lambda)$ is analytic on $\mathbf{C}^{+}$, then $\hat{L}(\alpha)$ is supported on $\mathbf{R}^{-}$, and hence the right-hand sides in (6.11), (6.12), (6.15), and (6.16) vanish when $x<0$. Since these equations are uniquely solvable, their solutions vanish as well, and therefore $k$ is supported on $\mathbf{R}^{+}$. We have thus proved the converse of Proposition 3.3.

It remains to prove that the potential $k(x)$ obtained by the Marchenko method has entries in $L^{1}(\mathbf{R})$. To do so, we modify the inversion procedure as follows. We solve one of the Marchenko equations (6.9) and (6.10) for $x>0$ and then employ (4.19) to compute $k(x)$ for $x>0$. By the same token, we solve one of (6.11) and (6.12) for $x<0$ and then 
use (4.19) to find $k(x)$ for $x<0$. In fact, this procedure will be implemented in the case of rational reflection coefficients in Section 8.

We first derive the following partial characterization result.

THEOREM 6.2. Let $R(\lambda)$ be a matrix function in $\mathcal{W}^{n}$ such that

$$
\sup _{\lambda \in \mathbf{R}}\|R(\lambda)\|<1, \quad \int_{0}^{\infty} d \alpha\left(\|\hat{R}(\alpha)\|+\alpha\|\hat{R}(\alpha)\|^{2}\right)<+\infty
$$

where $\hat{R}(\alpha)$ is defined in (6.4). Then, for $x>0$, the unique solutions $B_{l 2}(x, \alpha)$ and $B_{l 3}(x, \alpha)$ of (6.9) and (6.10), respectively, satisfy

$$
\int_{0}^{\infty} d x\left\|B_{l j}\left(x, 0^{+}\right)\right\|<+\infty, \quad j=2,3
$$

In particular, the entries of $k(x)=2 i B_{l 2}\left(x, 0^{+}\right)$and $k(x)=2 i B_{l 3}\left(x, 0^{+}\right)^{\dagger}$ belong to $L^{1}\left(\mathbf{R}^{+}\right)$. Similarly, let $L(\lambda)$ be a matrix function in $\mathcal{W}^{n}$ such that

$$
\sup _{\lambda \in \mathbf{R}}\|L(\lambda)\|<1, \quad \int_{-\infty}^{0} d \alpha\left(\|\hat{L}(\alpha)\|-\alpha\|\hat{L}(\alpha)\|^{2}\right)<+\infty
$$

where $\hat{L}(\alpha)$ is defined in (6.4). Then for $x<0$ the unique solutions $B_{r 2}(x, \alpha)$ and $B_{r 3}(x, \alpha)$ of (6.11) and (6.12), respectively, satisfy

$$
\int_{-\infty}^{0} d x\left\|B_{r j}\left(x, 0^{+}\right)\right\|<+\infty, \quad j=2,3 .
$$

In particular, the entries of $k(x)=-2 i B_{r 2}\left(x, 0^{+}\right)$and $k(x)=-2 i B_{r 3}\left(x, 0^{+}\right)^{\dagger}$ belong to $L^{1}\left(\mathbf{R}^{-}\right)$.

Proof. We only prove the theorem for $x>0$, as the proof for $x<0$ is similar. Put

$$
\hat{R}_{\Delta}(\alpha)=\left[\begin{array}{cc}
0 & -\hat{R}(\alpha) \\
-\hat{R}(\alpha)^{\dagger} & 0
\end{array}\right]
$$

and consider the integral equation

$$
B_{l}(x, \alpha)-\int_{0}^{\infty} d \beta B_{l}(x, \beta) \hat{R}_{\Delta}(2 x+\alpha+\beta)=\hat{R}_{\Delta}(2 x+\alpha), \quad \alpha>0
$$


Then its unique solution in $L^{1}\left(\mathbf{R}^{+} ; \mathbf{C}^{2 n \times 2 n}\right)$ coincides with the matrix function $B_{l}(x, \alpha)$ in (4.3) and the integral operator is a strict contraction on $L^{2}\left(\mathbf{R}^{+} ; \mathbf{C}^{2 n \times 2 n}\right)$. Iterating the adjoint of (6.20) we have

$$
B_{l}(x, \alpha)^{\dagger}=\sum_{j=0}^{\infty} B_{l}^{(j)}(x, \alpha)^{\dagger}
$$

where $B_{l}^{(0)}(x, \alpha)=\hat{R}_{\Delta}(2 x+\alpha)$ and

$$
B_{l}^{(j)}(x, \alpha)^{\dagger}=\int_{0}^{\infty} d \beta \hat{R}_{\Delta}(2 x+\alpha+\beta) B_{l}^{(j-1)}(x, \beta)^{\dagger}, \quad j \geq 1
$$

Now let $\rho \in[0,1)$ be the spectral radius of the integral operator appearing in (the adjoint of) (6.20). Then, by the selfadjointness of this operator on $L^{2}\left(\mathbf{R}^{+} ; \mathbf{C}^{2 n \times 2 n}\right)$ and the Schwarz inequality, we get

$$
\left\|B_{l}^{(j)}(x, \alpha)\right\| \leq \rho^{j-1} \int_{2 x}^{\infty} d \beta\left\|\hat{R}_{\Delta}(\beta)\right\|^{2}, \quad j \geq 1
$$

and therefore

$$
\begin{aligned}
\int_{0}^{\infty} d x\left\|B_{l}(x, \alpha)\right\| & \leq \frac{1}{2}\left[\int_{\alpha}^{\infty} d \beta\left\|\hat{R}_{\Delta}(\beta)\right\|+\frac{1}{1-\rho} \int_{0}^{\infty} d y \int_{y+\alpha}^{\infty} d \beta\left\|\hat{R}_{\Delta}(\beta)\right\|^{2}\right] \\
& \leq \frac{1}{2}\left[\int_{\alpha}^{\infty} d \beta\left\|\hat{R}_{\Delta}(\beta)\right\|+\frac{1}{1-\rho} \int_{\alpha}^{\infty} d \beta(\beta-\alpha)\left\|\hat{R}_{\Delta}(\beta)\right\|^{2}\right]
\end{aligned}
$$

which is finite.

Let us observe that (6.18) and (6.19) amount to requiring that the integral operators with integral kernels $\hat{R}(\alpha+\beta)^{\dagger}$ and $\hat{L}(-\alpha-\beta)$ have square integrable kernels and hence are Hilbert-Schmidt on $L^{2}\left(\mathbf{R}^{+} ; \mathbf{C}^{n}\right)$. Note also that rational matrix functions $R(\lambda)$ and $L(\lambda)$ without real poles that vanish at infinity satisfy the assumptions of Theorem 6.2. The identities [cf. (6.4) and (3.14)]

$$
\begin{gathered}
L(\lambda)=\int_{-\infty}^{\infty} d \alpha e^{i \lambda \alpha} \hat{L}(-\alpha), \quad R(\lambda)^{\dagger}=\int_{-\infty}^{\infty} d \alpha e^{i \lambda \alpha} \hat{R}(\alpha)^{\dagger}, \\
L(\lambda)=-T_{r}(\lambda) R(\lambda)^{\dagger}\left[T_{l}(\lambda)^{\dagger}\right]^{-1}, \quad \lambda \in \mathbf{R},
\end{gathered}
$$


in combination with the analyticity properties of $T_{r}(\lambda)$ and $T_{l}\left(\lambda^{*}\right)^{\dagger}$ and their inverses, imply that if one of the integral operators is Hilbert-Schmidt then so is the other. In other words, the conditions (6.18) and (6.19) are equivalent.

The natural conditions under which one expects to reconstruct a potential with $L^{1}$ entries for $x>0$ would be $R \in \mathcal{W}^{n}$ and

$$
\sup _{\lambda \in \mathbf{R}}\|R(\lambda)\|<1, \quad \lim _{\lambda \rightarrow \pm \infty}\|R(\lambda)\|=0 .
$$

However, evaluating the first iterate of $(6.20)$ as $\alpha \rightarrow 0^{+}$, i.e.,

$$
B_{l}^{(1)}\left(x, 0^{+}\right)=\int_{2 x}^{\infty} d \beta \hat{R}_{\Delta}(\beta)^{2}=\left[\begin{array}{cc}
\int_{2 x}^{\infty} d \beta \hat{R}(\beta) \hat{R}(\beta)^{\dagger} & 0 \\
0 & \int_{2 x}^{\infty} d \beta \hat{R}(\beta)^{\dagger} \hat{R}(\beta)
\end{array}\right]
$$

one sees that (6.18) is expected to hold if the integral $\int_{0}^{\infty} d x\left\|B_{l}^{(1)}\left(x, 0^{+}\right)\right\|$is to be finite.

Using the method of the proof of Theorem 2 of [34], one easily obtains the estimate

$$
\int_{2 x_{0}}^{\infty} d x\left\|B_{l}^{(2 j)}\left(x, 0^{+}\right)\right\| \leq \frac{1}{2}\left(\int_{2 x_{0}}^{\infty} d \beta\|\hat{R}(\beta)\|\right)^{j+1}, \quad j \geq 0
$$

where one assumes only that $\int_{0}^{\infty} d \alpha\|\hat{R}(\alpha)\|<+\infty$. Unfortunately, this estimate does not extend to the odd iterates of (6.20). However, since $\hat{R}_{\Delta}$ belongs to the class $\mathbf{K}_{\Delta}$ introduced in [34], it follows from this theorem that the potential $k(x)$ obtained has an $L^{1}$-tail in the sense that

$$
\exists x_{0}>0: \quad\left(\int_{-\infty}^{-x_{0}}+\int_{x_{0}}^{\infty}\right) d x\|k(x)\|<+\infty .
$$

Moreover, for every $\lambda \in \mathbf{R}$ the Jost solution $F_{l}(x, \lambda)$ is differentiable with respect to $x$ if $x>x_{0}$ and $F_{r}(x, \lambda)$ is differentiable with respect to $x$ for $x<-x_{0}$, thus providing a partial justification of (5.5). In other words, neither in the work of Melik-Adamyan [34] for the half line nor in the present work for the full line, a complete characterization is given of the scattering data leading to a unique $L^{1}$-potential. One does not obtain such a characterization either even if one combines Melik-Adamyan's reduction of the inverse 
problem on the full line to that on the half line [32] with his solution of the inverse problem on the half line [34].

\section{Construction of the Scattering Matrix}

We now show that the scattering matrix $\mathbf{S}(\lambda)$ can be constructed in terms of $L(\lambda)$ or $R(\lambda)$ alone. This can be seen as follows. Since the construction from $L(\lambda)$ is similar to that from $R(\lambda)$, we will only illustrate the latter. Given $R(\lambda)$ for $\lambda \in \mathbf{R}$, we can obtain $T_{l}(\lambda)$ by solving the matrix Riemann-Hilbert problem

$$
T_{l}(\lambda) T_{l}(\lambda)^{\dagger}=I_{n}-R(\lambda) R(\lambda)^{\dagger}, \quad \lambda \in \mathbf{R},
$$

which follows from (3.12). Here $T_{l}(\lambda)$ is continuous in $\overline{\mathbf{C}^{+}}$, is analytic in $\mathbf{C}^{+}$, and converges to $I_{n}$ as $\lambda \rightarrow \infty$ in $\overline{\mathbf{C}^{+}}$; similarly, $T_{l}\left(\lambda^{*}\right)^{\dagger}$ is continuous for $\lambda \in \overline{\mathbf{C}^{-}}$, is analytic for $\lambda \in \mathbf{C}^{-}$, and converges to $I_{n}$ as $\lambda \rightarrow \infty$ in $\overline{\mathbf{C}^{-}}$. In a similar way, $T_{r}(\lambda)$ can be constructed by solving the matrix Riemann-Hilbert problem

$$
T_{r}(\lambda)^{\dagger} T_{r}(\lambda)=I_{n}-R(\lambda)^{\dagger} R(\lambda), \quad \lambda \in \mathbf{R},
$$

which is found from (3.12). When the scattering coefficients are considered for complex $\lambda$, instead of (6.21) we must use

$$
L(\lambda)=-T_{r}(\lambda) R\left(\lambda^{*}\right)^{\dagger}\left[T_{l}\left(\lambda^{*}\right)^{\dagger}\right]^{-1}, \quad \lambda \in \mathbf{C},
$$

which follows with the help of Proposition 2.6. Once $T_{l}(\lambda)$ and $T_{r}(\lambda)$ are obtained, we can recover $L(\lambda)$ using (7.3). Note that from Theorem 4.2, it follows that the $n \times n$ matrices in the right-hand sides in (7.1) and (7.2) both belong to the Wiener algebra $\mathcal{W}^{n}$. Furthermore, from Corollary 3.2 and Theorem 4.2, it follows that $T_{l}(\lambda)$ and $T_{r}(\lambda)$ belong to the subalgebra $\mathcal{W}_{+}^{n}$, and $T_{l}\left(\lambda^{*}\right)^{\dagger}$ and $T_{r}\left(\lambda^{*}\right)^{\dagger}$ belong to $\mathcal{W}_{-}^{n}$. Hence, (7.1) and (7.2) lead us to a left and a right, respectively, canonical Wiener-Hopf factorization in $\mathcal{W}^{n}$. 
From the theory of transfer functions [13], since $R(\lambda) \rightarrow 0$ as $\lambda \rightarrow \pm \infty$, it follows that any rational $R(\lambda)$ can be represented in the form

$$
R(\lambda)=i \mathcal{C}(\lambda-i \mathcal{A})^{-1} \mathcal{B}, \quad \lambda \in \mathbf{C}
$$

where $\mathcal{A}, \mathcal{B}$, and $\mathcal{C}$ are independent of $\lambda$ and belong to $\mathbf{C}^{p \times p}, \mathbf{C}^{p \times n}$, and $\mathbf{C}^{n \times p}$, respectively, for some integer $p$. Here it is assumed that the order $p$ of $\mathcal{A}$ is minimal, i.e. the realization (7.4) is minimal and hence unique up to similarity.

Next, when $R(\lambda)$ is a rational function, we will construct $\mathbf{S}(\lambda)$ in terms of the matrices $\mathcal{A}, \mathcal{B}$, and $\mathcal{C}$ given in (7.4). Since $R(\lambda)$ is continuous for $\lambda \in \mathbf{R}$, from the minimality of the realization given in (7.4) it follows that $\mathcal{A}$ does not have any eigenvalues on the imaginary axis. Using (7.4) in (7.1) and (7.2), we obtain

$$
\begin{gathered}
T_{l}(\lambda) T_{l}\left(\lambda^{*}\right)^{\dagger}=I_{n}-i\left[\begin{array}{ll}
\mathcal{C} & 0
\end{array}\right]\left(\lambda-i \mathcal{K}_{l}\right)^{-1}\left[\begin{array}{c}
0 \\
\mathcal{C}^{\dagger}
\end{array}\right] \\
T_{r}\left(\lambda^{*}\right)^{\dagger} T_{r}(\lambda)=I_{n}+i\left[\begin{array}{ll}
0 & \mathcal{B}^{\dagger}
\end{array}\right]\left(\lambda-i \mathcal{K}_{r}\right)^{-1}\left[\begin{array}{c}
\mathcal{B} \\
0
\end{array}\right]
\end{gathered}
$$

where now $\lambda \in \mathbf{C}$ and we have defined

$$
\mathcal{K}_{l}=\left[\begin{array}{cc}
\mathcal{A} & -\mathcal{B B}^{\dagger} \\
0 & -\mathcal{A}^{\dagger}
\end{array}\right], \quad \mathcal{K}_{r}=\left[\begin{array}{cc}
\mathcal{A} & 0 \\
\mathcal{C}^{\dagger} \mathcal{C} & -\mathcal{A}^{\dagger}
\end{array}\right]
$$

Note that the inverses of the right-hand sides in (7.5) and (7.6) can be written as

$$
\begin{gathered}
{\left[T_{l}\left(\lambda^{*}\right)^{\dagger}\right]^{-1} T_{l}(\lambda)^{-1}=I_{n}+i\left[\begin{array}{ll}
\mathcal{C} & 0
\end{array}\right](\lambda-i \mathcal{E})^{-1}\left[\begin{array}{c}
0 \\
\mathcal{C}^{\dagger}
\end{array}\right]} \\
T_{r}(\lambda)^{-1}\left[T_{r}\left(\lambda^{*}\right)^{\dagger}\right]^{-1}=I_{n}-i\left[\begin{array}{ll}
0 & \mathcal{B}^{\dagger}
\end{array}\right](\lambda-i \mathcal{E})^{-1}\left[\begin{array}{c}
\mathcal{B} \\
0
\end{array}\right],
\end{gathered}
$$

where $\mathcal{E}$ is the "state characteristic matrix" given by

$$
\mathcal{E}=\left[\begin{array}{cc}
\mathcal{A} & -\mathcal{B B}^{\dagger} \\
\mathcal{C}^{\dagger} \mathcal{C} & -\mathcal{A}^{\dagger}
\end{array}\right]
$$

which, apart from some factors $i=\sqrt{-1}$, has been used in [27]. 
If $\mathcal{A}$ has all its eigenvalues in the left half plane, i.e. if $R(\lambda)$ is analytic in $\mathbf{C}^{+}$, then it is a simple procedure to obtain $T_{l}(\lambda), T_{r}(\lambda)$, and their inverses in terms of $\mathcal{A}, \mathcal{B}$, and $\mathcal{C}$. This is because certain solutions of some simple Riccati equations can be used to construct the so-called supporting projections in the realizations (7.5) and (7.6) and then to apply the factorization theory of [13]. When $\mathcal{A}$ has at least one eigenvalue in the right half plane, similarity transformations must first be applied to (7.5) and (7.6) to bring the similarity images of $\mathcal{K}_{l}$ and $\mathcal{K}_{r}$ in a form amenable to the same treatment as if $\mathcal{A}$ would have only eigenvalues in the left half plane.

Let us suppose that

$$
\mathcal{A}=\left[\begin{array}{cc}
\mathcal{A}_{-} & 0 \\
0 & \mathcal{A}_{+}
\end{array}\right]
$$

with respect to a suitable basis, where $\mathcal{A}_{-}$has order $p_{-}$and has all its eigenvalues in the left half plane and $\mathcal{A}_{+}$has order $p_{+}$and has all its eigenvalues in the right half plane. Here $p_{-}+p_{+}=p$. This form can always be achieved by means of a suitable similarity transformation and an appropriate transformation of the matrices $\mathcal{B}$ and $\mathcal{C}$. Put

$$
\Phi_{l}=\left[\begin{array}{cccc}
I_{p_{-}} & 0 & 0 & 0 \\
0 & 0 & 0 & I_{p_{+}} \\
0 & 0 & I_{p_{-}} & 0 \\
0 & -I_{p_{+}} & 0 & P_{1}
\end{array}\right], \quad \Phi_{r}=\left[\begin{array}{cccc}
I_{p_{-}} & 0 & 0 & 0 \\
0 & -P_{2} & 0 & I_{p_{+}} \\
0 & 0 & I_{p_{-}} & 0 \\
0 & -I_{p_{+}} & 0 & 0
\end{array}\right]
$$

where $P_{1}$ and $P_{2}$ will be specified shortly. Let us partition $\mathcal{B}$ and $\mathcal{C}$ as

$$
\mathcal{B}=\left[\begin{array}{l}
\mathcal{B}_{-} \\
\mathcal{B}_{+}
\end{array}\right], \quad \mathcal{C}=\left[\begin{array}{ll}
\mathcal{C}_{-} & \mathcal{C}_{+}
\end{array}\right]
$$

where $\mathcal{B}_{ \pm}$and $\mathcal{C}_{ \pm}$are $p_{ \pm} \times n$ and $n \times p_{ \pm}$matrices, respectively. We then take for $P_{1}$ and $P_{2}$ the unique solutions of the Lyapunov equations (cf. Theorem I 4.1 of [23], Theorem VII 2.4 of [17])

$$
\begin{aligned}
& \mathcal{A}_{+} P_{1}+P_{1} \mathcal{A}_{+}^{\dagger}=\mathcal{B}_{+} \mathcal{B}_{+}^{\dagger}, \\
& P_{2} \mathcal{A}_{+}+\mathcal{A}_{+}^{\dagger} P_{2}=\mathcal{C}_{+}^{\dagger} \mathcal{C}_{+} .
\end{aligned}
$$


In fact, we have

$$
P_{1}=\int_{0}^{\infty} d t e^{-t \mathcal{A}_{+}} \mathcal{B}_{+} \mathcal{B}_{+}^{\dagger} e^{-t \mathcal{A}_{+}^{\dagger}}, \quad P_{2}=\int_{0}^{\infty} d t e^{-t \mathcal{A}_{+}^{\dagger}} \mathcal{C}_{+}^{\dagger} \mathcal{C}_{+} e^{-t \mathcal{A}_{+}}
$$

so that $P_{1}$ and $P_{2}$ are positive selfadjoint. Then, we easily compute

$$
\Omega_{1}=\Phi_{l} \mathcal{K}_{l} \Phi_{l}^{-1}=\left[\begin{array}{cc}
\Omega_{3} & -\Omega_{5} \Omega_{5}^{\dagger} \\
0 & -\Omega_{3}^{\dagger}
\end{array}\right], \quad \Omega_{2}=\Phi_{r} \mathcal{K}_{r} \Phi_{r}^{-1}=\left[\begin{array}{cc}
\Omega_{4} & 0 \\
\Omega_{6}^{\dagger} \Omega_{6} & -\Omega_{4}^{\dagger}
\end{array}\right]
$$

where

$$
\Omega_{3}=\left[\begin{array}{cc}
\mathcal{A}_{-} & -\mathcal{B}_{-} \mathcal{B}_{+}^{\dagger} \\
0 & -\mathcal{A}_{+}^{\dagger}
\end{array}\right], \quad \Omega_{4}=\left[\begin{array}{cc}
\mathcal{A}_{-} & 0 \\
\mathcal{C}_{+}^{\dagger} \mathcal{C}_{-} & -\mathcal{A}_{+}^{\dagger}
\end{array}\right], \quad \Omega_{5}=\left[\begin{array}{c}
\mathcal{B}_{-} \\
0
\end{array}\right], \quad \Omega_{6}=\left[\begin{array}{ll}
\mathcal{C}_{-} & 0
\end{array}\right]
$$

Due to the contractivity of $R(\lambda)$ and $R(\lambda)^{\dagger}$ for $\lambda \in \mathbf{R}$ given in (6.17), we have the following result (Theorems 3.2 and 3.4 of [27]):

Proposition 7.1. Let $\mathcal{A}, \mathcal{B}$, and $\mathcal{C}$ be the matrices in the minimal realization given by (7.4) and consider the quadratic matrix equations

$$
\begin{aligned}
& \mathcal{A X}+\mathcal{X} \mathcal{A}^{\dagger}=\mathcal{B B}^{\dagger}+\mathcal{X} \mathcal{C}^{\dagger} \mathcal{C} \mathcal{X} \\
& \mathcal{A}^{\dagger} \mathcal{Y}+\mathcal{Y} \mathcal{A}=\mathcal{C}^{\dagger} \mathcal{C}+\mathcal{Y} \mathcal{B B}^{\dagger} \mathcal{Y}
\end{aligned}
$$

Then all hermitian solutions $\mathcal{X}$ of (7.13) are nonsingular, and the number of positive (resp. negative) eigenvalues of $\mathcal{X}$ coincides with the number of poles of $R(\lambda)$ in $\mathbf{C}^{+}\left(\right.$resp. in $\left.\mathbf{C}^{-}\right)$. There is at least one such solution $\mathcal{X}$. An analogous result holds for hermitian solutions of $(7.14)$

Since the order of $\mathcal{A}$ is minimal, the number of positive and negative eigenvalues of any hermitian solution $\mathcal{X}$ of (7.13) coincides with the number of eigenvalues of $\mathcal{A}$ in the right and left half plane, respectively. In counting the number of poles and eigenvalues, (algebraic) multiplicities have been taken into account. The nonlinear equations (7.13) and (7.14) are called state characteristic equations in [27] and Riccati equations elsewhere in the literature (e.g. [31]). 
The following result allows one to obtain explicit expressions for the factors $T_{l}(\lambda)$ and $T_{r}(\lambda)$ and their inverses.

THEOREM 7.2. Let $\mathcal{A}, \mathcal{B}$, and $\mathcal{C}$ be the matrices in the minimal realization given by (7.4). Then, there exist unique hermitian solutions $\mathcal{X}$ and $\mathcal{Y}$ of the state characteristic equations (7.13) and (7.14) such that the matrices

$$
\mathcal{E}_{r}=\mathcal{A}-\mathcal{B B}^{\dagger} \mathcal{Y}, \quad \mathcal{E}_{l}=\mathcal{A}-\mathcal{X} \mathcal{C}^{\dagger} \mathcal{C}
$$

have all their eigenvalues in the left half complex plane.

Proof. Let $\Upsilon=J_{2 p} \mathbf{q}_{2 p} \mathcal{E}$ and $\Xi=i J_{2 p} \mathbf{q}_{2 p}$. We first observe that $-i \mathcal{E}$ is both $\Upsilon$ selfadjoint and $\Xi$-selfadjoint, i.e. both $-i \Upsilon \mathcal{E}$ and $-i \Xi \mathcal{E}$ are selfadjoint. Then the spectral subspaces of $\mathcal{E}$ corresponding to the eigenvalues in the left and right half planes are both $\Upsilon$-neutral and $\Xi$-neutral, i.e., for any vector $u$ from such a spectral subspace we have

$$
\langle\Upsilon u, u\rangle=\langle\Xi u, u\rangle=0
$$

Following the proof of Lemma 7.2.2 of [31], let us write the spectral subspace of $\mathcal{E}$ corresponding to its eigenvalues in the left half plane in the form

$$
\mathcal{L}=\left\{\left[\begin{array}{l}
\mathcal{Y}_{l} \\
\mathcal{Y}_{r}
\end{array}\right] u: u \in \mathbf{C}^{p}\right\}
$$

where we note that $\mathcal{L}$ is $p$-dimensional, because of the similarity relation $J_{2 p} \mathbf{q}_{2 p} \mathcal{E} \mathbf{q}_{2 p} J_{2 p}=$ $-\mathcal{E}^{\dagger}$. Thus the $\mathcal{E}$-invariance of $\mathcal{L}$ leads to the two identities

$$
\begin{aligned}
\mathcal{A} \mathcal{Y}_{l}-\mathcal{B B} \mathcal{B}^{\dagger} \mathcal{Y}_{r} & =\mathcal{Y}_{l} \mathcal{G}, \\
\mathcal{C}^{\dagger} \mathcal{C} \mathcal{Y}_{l}-\mathcal{A}^{\dagger} \mathcal{Y}_{r} & =\mathcal{Y}_{r} \mathcal{G},
\end{aligned}
$$

where $\mathcal{G}$ is some $p \times p$ matrix. Then the $\Upsilon$-neutrality of $\mathcal{L}$ means that for every $u \in \mathbf{C}^{p}$ we get

$$
u^{\dagger}\left[\begin{array}{ll}
\mathcal{Y}_{l}^{\dagger} & \mathcal{Y}_{r}^{\dagger}
\end{array}\right] \Upsilon\left[\begin{array}{l}
\mathcal{Y}_{l} \\
\mathcal{Y}_{r}
\end{array}\right] u=u^{\dagger}\left(\mathcal{Y}_{l}^{\dagger} \mathcal{C}^{\dagger} \mathcal{C} \mathcal{Y}_{l}-\mathcal{Y}_{l}^{\dagger} \mathcal{A}^{\dagger} \mathcal{Y}_{r}-\mathcal{Y}_{r}^{\dagger} \mathcal{A} \mathcal{Y}_{l}+\mathcal{Y}_{r}^{\dagger} \mathcal{B} \mathcal{B}^{\dagger} \mathcal{Y}_{r}\right) u=0
$$


Hence, when $\mathcal{Y}_{l} u=0$, we have $\left\|\mathcal{B}^{\dagger} \mathcal{Y}_{r} u\right\|_{2}^{2}=0$, implying $\mathcal{B}^{\dagger} \mathcal{Y}_{r} u=0$. As a result of (7.16) we also have $\mathcal{Y}_{l} \mathcal{G} u=0$ whenever $\mathcal{Y}_{l} u=0$, and by virtue of $(7.17)$ we have

$$
\mathcal{A}^{\dagger} \mathcal{Y}_{r} u=-\mathcal{Y}_{r} \mathcal{G} u \in \mathcal{Y}_{r}\left[\operatorname{Ker} \mathcal{Y}_{l}\right]
$$

whenever $\mathcal{Y}_{l} u=0$, where $\operatorname{Ker} \mathcal{Y}_{l}$ denotes the null space of the matrix $\mathcal{Y}_{l}$. Thus the subspace $\mathcal{Y}_{r}\left[\operatorname{Ker} \mathcal{Y}_{l}\right]$ is invariant under $\mathcal{A}^{\dagger}$, while, as we have seen above, it is contained in $\operatorname{Ker} \mathcal{B}^{\dagger}$. In other words,

$$
\mathcal{Y}_{r}\left[\operatorname{Ker} \mathcal{Y}_{l}\right] \subset \bigcap_{r=0}^{\infty} \operatorname{Ker}\left(\mathcal{B}^{\dagger}\left(\mathcal{A}^{\dagger}\right)^{r}\right)=\{0\},
$$

due to the minimality of the realization given in (7.4). Consequently, if $\mathcal{Y}_{l} u=0$ then $\mathcal{Y}_{l} u=\mathcal{Y}_{r} u=0$, which leads to $u=0$ because $\operatorname{dim} \mathcal{L}=p$. In other words, we have proved the nonsingularity of $\mathcal{Y}_{l}$ and hence we may write $\mathcal{L}$ in the form

$$
\mathcal{L}=\left\{\left[\begin{array}{l}
I_{p} \\
\mathcal{Y}
\end{array}\right] u: u \in \mathbf{C}^{p}\right\}
$$

where $\mathcal{Y}=\mathcal{Y}_{r} \mathcal{Y}_{l}^{-1}$

In a similar way, let us write the spectral subspace of $\mathcal{E}$ corresponding to the eigenvalues in the right half plane as

$$
\mathcal{M}=\left\{\left[\begin{array}{l}
\mathcal{X}_{l} \\
\mathcal{X}_{r}
\end{array}\right] u: u \in \mathbf{C}^{p}\right\}
$$

where $\operatorname{dim} \mathcal{M}=p$. Then the $\mathcal{E}$-invariance of $\mathcal{M}$ leads to the two identities

$$
\begin{gathered}
\mathcal{A} \mathcal{X}_{l}-\mathcal{B B}^{\dagger} \mathcal{X}_{r}=\mathcal{X}_{l} \mathcal{H} \\
\mathcal{C}^{\dagger} \mathcal{C} \mathcal{X}_{l}-\mathcal{A}^{\dagger} \mathcal{X}_{r}=\mathcal{X}_{r} \mathcal{H}
\end{gathered}
$$

where $\mathcal{H}$ is some $p \times p$ matrix. Then the $\Upsilon$-neutrality of $\mathcal{M}$ means that for every $u \in \mathbf{C}^{p}$ the expression (7.18) with $\mathcal{Y}_{l}$ and $\mathcal{Y}_{r}$ replaced by $\mathcal{X}_{l}$ and $\mathcal{X}_{r}$ is valid. This implies that $\left\|\mathcal{C} \mathcal{X}_{l} u\right\|_{2}^{2}=0$ and hence $\mathcal{C} \mathcal{X}_{l} u=0$ whenever $\mathcal{X}_{r} u=0$. From (7.20) in combination with the 
above, it follows that $\mathcal{X}_{r} \mathcal{H} u=0$ whenever $\mathcal{X}_{r} u=0$. With the help of (7.19) it then follows that the subspace $\mathcal{X}_{l}\left[\operatorname{Ker} \mathcal{X}_{r}\right]$ is invariant under $\mathcal{A}$ and contained in $\operatorname{Ker} \mathcal{C}$. Therefore,

$$
\mathcal{X}_{l}\left[\operatorname{Ker} \mathcal{X}_{r}\right] \subset \bigcap_{r=0}^{\infty} \operatorname{Ker}\left(\mathcal{C} \mathcal{A}^{r}\right)=\{0\}
$$

due to the minimality of the realization given in (7.4). Consequently, if $\mathcal{X}_{r} u=0$ then $\mathcal{X}_{l} u=\mathcal{X}_{r} u=0$, which leads to $u=0$ because $\operatorname{dim} \mathcal{M}=p$. In other words, we have proved the nonsingularity of $\mathcal{X}_{r}$ and hence we may write

$$
\mathcal{M}=\left\{\left[\begin{array}{l}
\mathcal{X} \\
I_{p}
\end{array}\right] u: u \in \mathbf{C}^{p}\right\}
$$

where $\mathcal{X}=\mathcal{X}_{l} \mathcal{X}_{r}^{-1}$.

Since the subspaces $\mathcal{L}$ and $\mathcal{M}$ above have dimension $p$, the $2 p \times 2 p$ matrix

$$
\Sigma=\left[\begin{array}{ll}
I_{p} & \mathcal{X} \\
\mathcal{Y} & I_{p}
\end{array}\right]
$$

is nonsingular. Hence, both $I_{p}-\mathcal{X Y}$ and $I_{p}-\mathcal{Y} \mathcal{X}$ are nonsingular, and

$$
\Sigma^{-1}=\left[\begin{array}{cc}
\left(I_{p}-\mathcal{X} \mathcal{Y}\right)^{-1} & -\left(I_{p}-\mathcal{X Y Y}\right)^{-1} \mathcal{X} \\
-\left(I_{p}-\mathcal{Y} \mathcal{X}\right)^{-1} \mathcal{Y} & \left(I_{p}-\mathcal{Y} \mathcal{X}\right)^{-1}
\end{array}\right]
$$

Using the state characteristic equations (7.13) and (7.14) and passing to the resolvents, we obtain

$$
(\lambda-i \mathcal{E})^{-1}=\Sigma\left[\begin{array}{cc}
\left(\lambda-i \mathcal{E}_{r}\right)^{-1} & 0 \\
0 & \left(\lambda+i \mathcal{E}_{l}^{\dagger}\right)^{-1}
\end{array}\right] \Sigma^{-1},
$$

where $\mathcal{E}_{r}$ and $\mathcal{E}_{l}$ are given by (7.15). Thus $\mathcal{E}_{r}$ and $\mathcal{E}_{l}$ have all their eigenvalues in the left half plane.

Before applying the main factorization result from [13] to (7.5) and (7.6), we need the following proposition ([36], Theorem 6.5 .3 of [31]). Recall that $\mathcal{K}_{l}$ and $\mathcal{K}_{r}$ are the matrices defined in (7.7).

Proposition 7.3. Let $\mathcal{M}$ be the invariant subspace of $\mathcal{E}$ corresponding to the eigenvalues in the left (resp. right) half plane and $\mathcal{N}$ be the invariant subspace of $\mathcal{K}_{r}$ (resp. $\left.\mathcal{K}_{l}\right)$ corresponding to its eigenvalues in the right (resp. left) half plane. Then $\mathcal{M} \oplus \mathcal{N}=\mathbf{C}^{2 p}$. 
Now let $\Pi$ be the projection whose range is the invariant subspace of $\mathcal{E}$ corresponding to the eigenvalues in the left half plane and whose kernel is the invariant subspace of $\mathcal{K}_{r}$ corresponding to its eigenvalues in the right half plane, and let $\mathcal{Q}$ be the projection whose range is the invariant subspace of $\mathcal{K}_{l}$ corresponding to its eigenvalues in the left half plane and whose kernel is the invariant subspace of $\mathcal{E}$ corresponding to its eigenvalues in the right half plane. Applying the main factorization result in [13], we obtain the following expressions for the transmission coefficients in terms of the matrices appearing in (7.4):

$$
\begin{gathered}
T_{r}\left(\lambda^{*}\right)^{\dagger}=I_{n}+i\left[\begin{array}{ll}
0 & \mathcal{B}^{\dagger}
\end{array}\right]\left(\lambda-i \mathcal{K}_{r}\right)^{-1}\left(I_{2 p}-\Pi\right)\left[\begin{array}{c}
\mathcal{B} \\
0
\end{array}\right], \\
T_{r}(\lambda)=I_{n}+i\left[\begin{array}{ll}
0 & \mathcal{B}^{\dagger}
\end{array}\right] \Pi\left(\lambda-i \mathcal{K}_{r}\right)^{-1}\left[\begin{array}{c}
\mathcal{B} \\
0
\end{array}\right], \\
T_{r}(\lambda)^{-1}=I_{n}-i\left[\begin{array}{ll}
0 & \mathcal{B}^{\dagger}
\end{array}\right](\lambda-i \mathcal{E})^{-1} \Pi\left[\begin{array}{c}
\mathcal{B} \\
0
\end{array}\right], \\
{\left[T_{r}\left(\lambda^{*}\right)^{\dagger}\right]^{-1}=I_{n}-i\left[\begin{array}{ll}
0 & \mathcal{B}^{\dagger}
\end{array}\right]\left(I_{2 p}-\Pi\right)(\lambda-i \mathcal{E})^{-1}\left[\begin{array}{c}
\mathcal{B} \\
0
\end{array}\right],} \\
T_{l}(\lambda)=I_{n}-i\left[\begin{array}{ll}
\mathcal{C} & 0
\end{array}\right]\left(\lambda-i \mathcal{K}_{l}\right)^{-1} \mathcal{Q}\left[\begin{array}{c}
0 \\
\mathcal{C}^{\dagger}
\end{array}\right], \\
T_{l}\left(\lambda^{*}\right)^{\dagger}=I_{n}-i\left[\begin{array}{ll}
\mathcal{C} & 0
\end{array}\right]\left(I_{2 p}-\mathcal{Q}\right)\left(\lambda-i \mathcal{K}_{l}\right)^{-1}\left[\begin{array}{c}
0 \\
\mathcal{C}^{\dagger}
\end{array}\right], \\
{\left[T_{l}\left(\lambda^{*}\right)^{\dagger}\right]^{-1}=I_{n}+i\left[\begin{array}{ll}
\mathcal{C} & 0
\end{array}\right](\lambda-i \mathcal{E})^{-1}\left(I_{2 p}-\mathcal{Q}\right)\left[\begin{array}{c}
0 \\
\mathcal{C}^{\dagger}
\end{array}\right],} \\
T_{l}(\lambda)^{-1}=I_{n}+i\left[\begin{array}{ll}
\mathcal{C} & 0
\end{array}\right] \mathcal{Q}(\lambda-i \mathcal{E})^{-1}\left[\begin{array}{c}
0 \\
\mathcal{C}^{\dagger}
\end{array}\right] .
\end{gathered}
$$

When $\mathcal{A}$ has all its eigenvalues in the left half plane, the projections $\Pi$ and $\mathcal{Q}$ are given by

$$
\Pi=\left[\begin{array}{ll}
I_{p} & 0 \\
\mathcal{Y} & 0
\end{array}\right], \quad \mathcal{Q}=\left[\begin{array}{cc}
I_{p} & -\mathcal{X} \\
0 & 0
\end{array}\right] .
$$

Then, using $\mathcal{X}$ and $\mathcal{Y}$ specified in Theorem 7.2, with the help of the identity

$$
\left(I_{p}-\mathcal{X Y}\right) \mathcal{E}_{r}=\mathcal{E}_{l}\left(I_{p}-\mathcal{X Y}\right)
$$


we can simplify (7.22)-(7.29) considerably. In fact, we get

$$
\begin{cases}T_{r}\left(\lambda^{*}\right)^{\dagger}=I_{n}-i \mathcal{B}^{\dagger}\left(\lambda+i \mathcal{A}^{\dagger}\right)^{-1} \mathcal{Y} \mathcal{B}, & T_{r}(\lambda)^{-1}=I_{n}-i \mathcal{B}^{\dagger} \mathcal{Y}\left(\lambda-i \mathcal{E}_{r}\right)^{-1} \mathcal{B}, \\ T_{r}(\lambda)=I_{n}+i \mathcal{B}^{\dagger} \mathcal{Y}(\lambda-i \mathcal{A})^{-1} \mathcal{B}, & {\left[T_{r}\left(\lambda^{*}\right)^{\dagger}\right]^{-1}=I_{n}+i \mathcal{B}^{\dagger}\left(\lambda+i \mathcal{E}_{r}^{\dagger}\right)^{-1} \mathcal{Y} \mathcal{B},} \\ T_{l}(\lambda)=I_{n}+i \mathcal{C}(\lambda-i \mathcal{A})^{-1} \mathcal{X} \mathcal{C}^{\dagger}, & {\left[T_{l}\left(\lambda^{*}\right)^{\dagger}\right]^{-1}=I_{n}+i \mathcal{C X}\left(\lambda+i \mathcal{E}_{l}^{\dagger}\right)^{-1} \mathcal{C}^{\dagger},} \\ T_{l}\left(\lambda^{*}\right)^{\dagger}=I_{n}-i \mathcal{C X}\left(\lambda+i \mathcal{A}^{\dagger}\right)^{-1} \mathcal{C}^{\dagger}, & T_{l}(\lambda)^{-1}=I_{n}-i \mathcal{C}\left(\lambda-i \mathcal{E}_{l}\right)^{-1} \mathcal{X} \mathcal{C}^{\dagger}\end{cases}
$$

The eight matrix functions in (7.31) have the analyticity properties stated in Corollary 3.2 because $\mathcal{E}_{r}$ and $\mathcal{E}_{l}$ have their eigenvalues in the left half plane as assured by Theorem 7.2. Moreover, from (7.13) and (7.14) we find that $T_{r}(\lambda)$ and $T_{l}(\lambda)$ satisfy (7.5) and (7.6), respectively. Using (7.31) and the identity

$$
i \mathcal{C}^{\dagger} \mathcal{C} \mathcal{X}=\left(\lambda+i \mathcal{A}^{\dagger}\right)-\left(\lambda+i \mathcal{E}_{l}^{\dagger}\right)
$$

we obtain

$$
-R\left(\lambda^{*}\right)^{\dagger}\left[T_{l}\left(\lambda^{*}\right)^{\dagger}\right]^{-1}=i \mathcal{B}^{\dagger}\left(\lambda+i \mathcal{E}_{l}^{\dagger}\right)^{-1} \mathcal{C}^{\dagger}
$$

Next, using (7.3), (7.13), (7.31), (7.33), and the identity

$$
i \mathcal{B B}^{\dagger}=\mathcal{X}\left(\lambda+i \mathcal{E}_{l}^{\dagger}\right)-(\lambda-i \mathcal{A}) \mathcal{X}
$$

we get

$$
L(\lambda)=i \mathcal{B}^{\dagger} \mathcal{Y}(\lambda-i \mathcal{A})^{-1} \mathcal{X} \mathcal{C}^{\dagger}+i \mathcal{B}^{\dagger}\left(I_{p}-\mathcal{Y} \mathcal{X}\right)\left(\lambda+i \mathcal{E}_{l}^{\dagger}\right)^{-1} \mathcal{C}^{\dagger}
$$

and hence our construction of $\mathbf{S}(\lambda)$ is complete when $\mathcal{A}$ has all its eigenvalues in the left half plane.

Let us now consider the most general situation in which $\mathcal{A}$ may also have eigenvalues in the right half plane. In that case, for the projection operators $\mathcal{Q}$ and $\Pi$ we obtain

$$
\begin{array}{ll}
\operatorname{Im} \mathcal{Q}=\Phi_{l}^{-1}\left[\mathbf{C}^{p} \oplus\{0\}\right], & \operatorname{Ker} \mathcal{Q}=\operatorname{Im}\left[\begin{array}{c}
\mathcal{X} \\
I_{p}
\end{array}\right], \\
\operatorname{Ker} \Pi=\Phi_{r}^{-1}\left[\{0\} \oplus \mathbf{C}^{p}\right], & \operatorname{Im} \Pi=\operatorname{Im}\left[\begin{array}{c}
I_{p} \\
\mathcal{Y}
\end{array}\right] .
\end{array}
$$


Considering the matrices $\Phi_{l}$ and $\Phi_{r}$ defined in (7.9) and partitioning their inverses into $p \times p$ blocks as

$$
\Phi_{l}^{-1}=\Lambda_{l}=\left[\begin{array}{cc}
\Lambda_{l 1} & \Lambda_{l 2} \\
\Lambda_{l 3} & \Lambda_{l 4}
\end{array}\right], \quad \Phi_{r}^{-1}=\Lambda_{r}=\left[\begin{array}{cc}
\Lambda_{r 1} & \Lambda_{r 2} \\
\Lambda_{r 3} & \Lambda_{r 4}
\end{array}\right],
$$

we easily find the expressions

$$
\begin{gathered}
\mathcal{Q}=\left[\begin{array}{ll}
\Lambda_{l 1}\left(\Lambda_{l 1}-\mathcal{X} \Lambda_{l 3}\right)^{-1} & -\Lambda_{l 1}\left(\Lambda_{l 1}-\mathcal{X} \Lambda_{l 3}\right)^{-1} \mathcal{X} \\
\Lambda_{l 3}\left(\Lambda_{l 1}-\mathcal{X} \Lambda_{l 3}\right)^{-1} & -\Lambda_{l 3}\left(\Lambda_{l 1}-\mathcal{X} \Lambda_{l 3}\right)^{-1} \mathcal{X}
\end{array}\right], \\
I_{2 p}-\Pi=\left[\begin{array}{ll}
-\Lambda_{r 2}\left(\Lambda_{r 4}-\mathcal{Y} \Lambda_{r 2}\right)^{-1} \mathcal{Y} & \Lambda_{r 2}\left(\Lambda_{r 4}-\mathcal{Y} \Lambda_{r 2}\right)^{-1} \\
-\Lambda_{r 4}\left(\Lambda_{r 4}-\mathcal{Y} \Lambda_{r 2}\right)^{-1} \mathcal{Y} & \Lambda_{r 4}\left(\Lambda_{r 4}-\mathcal{Y} \Lambda_{r 2}\right)^{-1}
\end{array}\right],
\end{gathered}
$$

where the inverses of $\Lambda_{l 1}-\mathcal{X} \Lambda_{l 3}$ and $\Lambda_{r 4}-\mathcal{Y} \Lambda_{r 2}$ exist, because $\operatorname{Im} \mathcal{Q} \oplus \operatorname{Ker} \mathcal{Q}=\mathbf{C}^{2 p}$ and $\operatorname{Im} \Pi \oplus \operatorname{Ker} \Pi=\mathbf{C}^{2 p}$.

We will now derive the analogs of the expressions in (7.31). Using (7.21), (7.29), (7.30), and (7.36), we get

$$
T_{l}(\lambda)^{-1}=I_{n}-i \mathcal{C} \Lambda_{l 1}\left(\Lambda_{l 1}-\mathcal{X} \Lambda_{l 3}\right)^{-1}\left(\lambda-i \mathcal{E}_{l}\right)^{-1} \mathcal{X} \mathcal{C}^{\dagger} .
$$

With the help of (7.21), (7.28), and (7.36) we derive

$$
\left[T_{l}\left(\lambda^{*}\right)^{\dagger}\right]^{-1}=I_{n}+i \mathcal{C X}\left(\lambda+i \mathcal{E}_{l}^{\dagger}\right)^{-1} \mathcal{J}_{1} \mathcal{C}^{\dagger}
$$

where

$$
\mathcal{J}_{1}=\left(I_{p}-\mathcal{Y} \mathcal{X}\right)^{-1}\left[I_{p}+\left(\Lambda_{l 3}-\mathcal{Y} \Lambda_{l 1}\right)\left(\Lambda_{l 1}-\mathcal{X} \Lambda_{l 3}\right)^{-1} \mathcal{X}\right]
$$

Using (7.21), (7.24), and (7.37) we obtain

$$
T_{r}(\lambda)^{-1}=I_{n}-i \mathcal{B}^{\dagger} \mathcal{Y}\left(\lambda-i \mathcal{E}_{r}\right)^{-1} \mathcal{J}_{2} \mathcal{B}
$$

where

$$
\mathcal{J}_{2}=\left(I_{p}-\mathcal{X Y}\right)^{-1}\left[I_{p}+\left(\Lambda_{r 2}-\mathcal{X} \Lambda_{r 4}\right)\left(\Lambda_{r 4}-\mathcal{Y} \Lambda_{r 2}\right)^{-1} \mathcal{Y}\right]
$$

Next, using (7.21), (7.25), (7.30), and (7.37), we get

$$
\left[T_{r}\left(\lambda^{*}\right)^{\dagger}\right]^{-1}=I_{n}+i \mathcal{B}^{\dagger} \Lambda_{r 4}\left(\Lambda_{r 4}-\mathcal{Y} \Lambda_{r 2}\right)^{-1}\left(\lambda+i \mathcal{E}_{r}^{\dagger}\right)^{-1} \mathcal{Y} \mathcal{B}
$$


From (7.12), (7.26), (7.35), (7.36), and the equality

$$
\Phi_{l} \mathcal{Q}\left[\begin{array}{c}
0 \\
\mathcal{C}^{\dagger}
\end{array}\right]=-\left[\begin{array}{c}
I_{p} \\
0
\end{array}\right]\left(\Lambda_{l 1}-\mathcal{X} \Lambda_{l 3}\right)^{-1} \mathcal{X C} \mathcal{C}^{\dagger}
$$

we obtain

$$
T_{l}(\lambda)=I_{n}+i \mathcal{C} \Lambda_{l 1}\left(\lambda-i \Omega_{3}\right)^{-1}\left(\Lambda_{l 1}-\mathcal{X} \Lambda_{l 3}\right)^{-1} \mathcal{X} \mathcal{C}^{\dagger} .
$$

Let us partition $\Phi_{l}$ and $\Phi_{r}$ defined in (7.9) into $p \times p$ blocks as

$$
\Phi_{l}=\left[\begin{array}{ll}
\Phi_{l 1} & \Phi_{l 2} \\
\Phi_{l 3} & \Phi_{l 4}
\end{array}\right], \quad \Phi_{r}=\left[\begin{array}{ll}
\Phi_{r 1} & \Phi_{r 2} \\
\Phi_{r 3} & \Phi_{r 4}
\end{array}\right] .
$$

Using (7.12), (7.27), (7.35), (7.36), as well as the identity

$$
\left[\begin{array}{ll}
\mathcal{C} & 0
\end{array}\right]\left(I_{2 p}-\mathcal{Q}\right) \Phi_{l}^{-1}=\mathcal{C} \mathcal{J}_{3}\left[\begin{array}{ll}
0 & I_{p}
\end{array}\right],
$$

where

$$
\mathcal{J}_{3}=\Lambda_{l 2}-\Lambda_{l 1}\left(\Lambda_{l 1}-\mathcal{X} \Lambda_{l 3}\right)^{-1}\left(\Lambda_{l 2}-\mathcal{X} \Lambda_{l 4}\right),
$$

we obtain

$$
T_{l}\left(\lambda^{*}\right)^{\dagger}=I_{n}-i \mathcal{C} \mathcal{J}_{3}\left(\lambda+i \Omega_{3}^{\dagger}\right)^{-1} \Phi_{l 4} \mathcal{C}^{\dagger} .
$$

With the help of (7.12), (7.23), (7.35), (7.37), and the identity

$$
\left[\begin{array}{ll}
0 & \mathcal{B}^{\dagger}
\end{array}\right] \Pi \Phi_{r}^{-1}=\mathcal{B}^{\dagger} \mathcal{J}_{4}\left[\begin{array}{ll}
I_{p} & 0
\end{array}\right]
$$

where

$$
\mathcal{J}_{4}=\Lambda_{r 3}-\Lambda_{r 4}\left(\Lambda_{r 4}-\mathcal{Y} \Lambda_{r 2}\right)^{-1}\left(\Lambda_{r 3}-\mathcal{Y} \Lambda_{r 1}\right),
$$

we get

$$
T_{r}(\lambda)=I_{n}+i \mathcal{B}^{\dagger} \mathcal{J}_{4}\left(\lambda-i \Omega_{4}\right)^{-1} \Phi_{r 1} \mathcal{B}
$$

Using (7.12), (7.22), (7.35), (7.37), as well as the identity

$$
\Phi_{r}\left(I_{2 p}-\Pi\right)\left[\begin{array}{c}
\mathcal{B} \\
0
\end{array}\right]=-\left[\begin{array}{c}
0 \\
I_{p}
\end{array}\right]\left(\Lambda_{r 4}-\mathcal{Y} \Lambda_{r 2}\right)^{-1} \mathcal{Y B},
$$


we are led to

$$
T_{r}\left(\lambda^{*}\right)^{\dagger}=I_{n}-i \mathcal{B}^{\dagger} \Lambda_{r 4}\left(\lambda+i \Omega_{4}^{\dagger}\right)^{-1}\left(\Lambda_{r 4}-\mathcal{Y} \Lambda_{r 2}\right)^{-1} \mathcal{Y} \mathcal{B}
$$

It is easily verified that the expressions in (7.38)-(7.45) have the correct analyticity properties indicated in Corollary 3.2 .

From (7.4), (7.32), (7.39), and $I_{p}-\mathcal{J}_{1}=-\Lambda_{l 3}\left(\Lambda_{l 1}-\mathcal{X} \Lambda_{l 3}\right)^{-1} \mathcal{X}$, we get

$$
-R\left(\lambda^{*}\right)^{\dagger}\left[T_{l}\left(\lambda^{*}\right)^{\dagger}\right]^{-1}=-i \mathcal{B}^{\dagger}\left(\lambda+i \mathcal{A}^{\dagger}\right)^{-1} \Lambda_{l 3}\left(\Lambda_{l 1}-\mathcal{X} \Lambda_{l 3}\right)^{-1} \mathcal{X} \mathcal{C}^{\dagger}+i \mathcal{B}^{\dagger}\left(\lambda+i \mathcal{E}_{l}^{\dagger}\right)^{-1} \mathcal{J}_{1} \mathcal{C}^{\dagger}
$$

Using (7.3), (7.44), (7.46), and some standard results on realizations (Chapter 1 of [13]), we obtain

$$
L(\lambda)=i \Omega_{8}\left(\lambda-i \Omega_{7}\right)^{-1} \Omega_{9}
$$

where

$$
\begin{gathered}
\Omega_{7}=\left[\begin{array}{ccc}
\Omega_{4} & -\Phi_{r 1} \mathcal{B B}^{\dagger} & \Phi_{r 1} \mathcal{B} \mathcal{B}^{\dagger} \\
0 & -\mathcal{A}^{\dagger} & 0 \\
0 & 0 & -\mathcal{E}_{l}^{\dagger}
\end{array}\right], \\
\Omega_{8}=\mathcal{B}^{\dagger}\left[\begin{array}{ccc}
\mathcal{J}_{4} & -I_{p} & I_{p}
\end{array}\right], \quad \Omega_{9}=\left[\begin{array}{c}
0 \\
\Lambda_{l 3}\left(\Lambda_{l 1}-\mathcal{X} \Lambda_{l 3}\right)^{-1} \mathcal{X} \\
\mathcal{J}_{1}
\end{array}\right] \mathcal{C}^{\dagger} .
\end{gathered}
$$

The realization (7.47) will in general not be minimal.

To simplify the realization of $L(\lambda)$ given in (7.47), we let

$$
\widetilde{\mathcal{A}}=\Psi \Omega_{7} \Psi^{-1}, \quad \widetilde{\mathcal{C}}=\Omega_{8} \Psi^{-1}, \quad \widetilde{\mathcal{B}}=\Psi \Omega_{9},
$$

so that

$$
L(\lambda)=i \widetilde{\mathcal{C}}(\lambda-i \widetilde{\mathcal{A}})^{-1} \widetilde{\mathcal{B}}
$$

Here $\Psi$ is a suitable similarity and we partition the matrices $\widetilde{\mathcal{A}}, \widetilde{\mathcal{B}}$, and $\widetilde{\mathcal{C}}$ by breaking up the middle blocks of order $p$ into blocks of order $p_{-}$and $p_{+}$. Further, we decompose the solution $\mathcal{X}$ of (7.13) as

$$
\mathcal{X}=\left[\begin{array}{ll}
\mathcal{X}_{1} & \mathcal{X}_{2} \\
\mathcal{X}_{3} & \mathcal{X}_{4}
\end{array}\right]
$$


so that $\mathcal{X}_{1}$ and $\mathcal{X}_{4}$ are selfadjoint and have orders $p_{-}$and $p_{+}$, respectively, and $\mathcal{X}_{2}^{\dagger}=\mathcal{X}_{3}$. Moreover, for one of the blocks of $\Omega_{9}$ we can write

$$
\begin{aligned}
\Lambda_{l 3}\left(\Lambda_{l 1}-\mathcal{X} \Lambda_{l 3}\right)^{-1} \mathcal{X} & =\left[\begin{array}{cc}
0 & 0 \\
0 & I_{p_{+}}
\end{array}\right]\left[\begin{array}{cc}
I_{p_{-}} & -\mathcal{X}_{2} \\
0 & P_{1}-\mathcal{X}_{4}
\end{array}\right]^{-1}\left[\begin{array}{cc}
\mathcal{X}_{1} & \mathcal{X}_{2} \\
\mathcal{X}_{3} & \mathcal{X}_{4}
\end{array}\right] \\
& =\left[\begin{array}{cc}
0 & 0 \\
\left(P_{1}-\mathcal{X}_{4}\right)^{-1} \mathcal{X}_{3} & \left(P_{1}-\mathcal{X}_{4}\right)^{-1} \mathcal{X}_{4}
\end{array}\right]
\end{aligned}
$$

Then defining the similarity $\Psi$ by

$$
\Psi=\left[\begin{array}{cccc}
0 & 0 & 0 & I_{p} \\
0 & I_{p_{-}} & 0 & 0 \\
0 & 0 & I_{p_{+}} & 0 \\
-I_{p} & P_{4} & 0 & P_{3}
\end{array}\right], \quad \Psi^{-1}=\left[\begin{array}{cccc}
P_{3} & P_{4} & 0 & -I_{p} \\
0 & I_{p_{-}} & 0 & 0 \\
0 & 0 & I_{p_{+}} & 0 \\
I_{p} & 0 & 0 & 0
\end{array}\right]
$$

where

$$
P_{3}=\int_{0}^{\infty} d t e^{t \Omega_{4}} \Phi_{r 1} \mathcal{B} \mathcal{B}^{\dagger} e^{t \mathcal{E}_{l}^{\dagger}}
$$

is the unique (and generally nonselfadjoint) solution of the Lyapunov equation

$$
\Omega_{4} P_{3}+P_{3} \mathcal{E}_{l}^{\dagger}=-\Phi_{r 1} \mathcal{B B}^{\dagger}
$$

and

$$
P_{4}=-\int_{0}^{\infty} d t e^{t \Omega_{4}} \Phi_{r 1} \mathcal{B B}_{-}^{\dagger} e^{t \mathcal{A}_{-}^{\dagger}}
$$

is the unique (and generally nonsquare matrix) solution of the Lyapunov equation

$$
\Omega_{4} P_{4}+P_{4} \mathcal{A}_{-}^{\dagger}=\Phi_{r 1} \mathcal{B B}_{-}^{\dagger}
$$

we arrive at

$$
\widetilde{\mathcal{A}}=\left[\begin{array}{cc}
\widetilde{\mathcal{A}}_{+} & 0 \\
0 & \widetilde{\mathcal{A}}_{-}
\end{array}\right], \quad \widetilde{\mathcal{B}}=\left[\begin{array}{c}
\widetilde{\mathcal{B}}_{+} \\
\widetilde{\mathcal{B}}_{-}
\end{array}\right], \quad \widetilde{\mathcal{C}}=\left[\begin{array}{ll}
\widetilde{\mathcal{C}}_{+} & \widetilde{\mathcal{C}}_{-}
\end{array}\right]
$$

where

$$
\tilde{\mathcal{A}}_{+}=\left[\begin{array}{cc}
-\mathcal{E}_{l}^{\dagger} & 0 \\
0 & -\mathcal{A}_{-}^{\dagger}
\end{array}\right], \quad \widetilde{\mathcal{A}}_{-}=\left[\begin{array}{cc}
-\mathcal{A}_{+}^{\dagger} & 0 \\
\Phi_{r 1} \mathcal{B B}_{+}^{\dagger} & \Omega_{4}
\end{array}\right]
$$




$$
\begin{aligned}
& \widetilde{\mathcal{B}}_{+}=\left[\begin{array}{c}
\mathcal{J}_{1} \mathcal{C}^{\dagger} \\
0
\end{array}\right], \quad \widetilde{\mathcal{B}}_{-}=\left[\begin{array}{c}
\left(P_{1}-\mathcal{X}_{4}\right)^{-1}\left(\mathcal{X}_{3} \mathcal{C}_{-}^{\dagger}+\mathcal{X}_{4} \mathcal{C}_{+}^{\dagger}\right) \\
P_{3} \mathcal{J}_{1} \mathcal{C}_{+}^{\dagger}
\end{array}\right] \\
& \widetilde{\mathcal{C}_{+}}=\left[\begin{array}{lll}
\mathcal{B}^{\dagger}\left(I_{p}+\mathcal{J}_{4} P_{3}\right) & \mathcal{B}^{\dagger} \mathcal{J}_{4} P_{4}-\mathcal{B}_{-}^{\dagger}
\end{array}\right], \quad \widetilde{\mathcal{C}}_{-}=\left[\begin{array}{ll}
-\mathcal{B}_{+}^{\dagger} & -\mathcal{B}^{\dagger} \mathcal{J}_{4}
\end{array}\right] .
\end{aligned}
$$

\section{Inverse Problem with Rational Scattering Matrices}

Let $R(\lambda)$ have the form (7.4) for certain matrices $\mathcal{A}, \mathcal{B}$, and $\mathcal{C}$, where $\mathcal{A}$ has the minimal order and hence does not have zero or purely imaginary eigenvalues. Then

$$
R(\lambda)=i \mathcal{C}(\lambda-i \mathcal{A})^{-1} \mathcal{B}=-\int_{-\infty}^{\infty} d t e^{-i \lambda t} \mathcal{C} E(t ;-\mathcal{A}) \mathcal{B}
$$

where

$$
E(t ;-\mathcal{A})=\left\{\begin{aligned}
e^{-t \mathcal{A}} P_{\mathcal{A}}^{(+)}=\frac{1}{2 \pi i} \int_{\Gamma_{+}} d z e^{-t z}(z-\mathcal{A})^{-1}, & t>0, \\
-e^{-t \mathcal{A}} P_{\mathcal{A}}^{(-)}=-\frac{1}{2 \pi i} \int_{\Gamma_{-}} d z e^{-t z}(z-\mathcal{A})^{-1}, & t<0,
\end{aligned}\right.
$$

is the bisemigroup generated by $\mathcal{A}$ (cf. $[14,15])$. Here $\Gamma_{+}$and $\Gamma_{-}$are the positively oriented simple Jordan contours in the right and left half plane enclosing all of the eigenvalues of $\mathcal{A}$ in the open right and left half plane, respectively, and $P_{\mathcal{A}}^{(+)}$and $P_{\mathcal{A}}^{(-)}$are the spectral projections of $\mathcal{A}$ corresponding to its eigenvalues in the right and left half plane, respectively.

Let us solve the Marchenko equation (6.10), where

$$
\hat{R}(t)=-\mathcal{C} E(t ;-\mathcal{A}) \mathcal{B}, \quad \hat{R}(t)^{\dagger}=-\mathcal{B}^{\dagger} E\left(t ;-\mathcal{A}^{\dagger}\right) \mathcal{C}^{\dagger}
$$

which are obtained from (6.4) and (8.1). Introducing the positive selfadjoint $p \times p$ matrices

$$
\mathcal{D}_{1}=\int_{0}^{\infty} d t E(t ;-\mathcal{A}) \mathcal{B} \mathcal{B}^{\dagger} E\left(t ;-\mathcal{A}^{\dagger}\right), \quad \mathcal{D}_{2}=\int_{0}^{\infty} d t E\left(t ;-\mathcal{A}^{\dagger}\right) \mathcal{C}^{\dagger} \mathcal{C} E(t ;-\mathcal{A})
$$

we obtain for $x>0$ the hermitian integral kernel of (6.10) as

$$
\int_{0}^{\infty} d \beta \hat{R}(\gamma+\beta+2 x) \hat{R}(\alpha+\beta+2 x)^{\dagger}=\mathcal{C} E(\gamma+2 x ;-\mathcal{A}) \mathcal{D}_{1} E\left(\alpha+2 x ;-\mathcal{A}^{\dagger}\right) \mathcal{C}^{\dagger}
$$


The unique solution of the separable integral equation (6.10) is then given by

$$
B_{l 3}(x, \alpha)=\mathcal{B}^{\dagger}\left[I_{p}-E\left(2 x ;-\mathcal{A}^{\dagger}\right) \mathcal{D}_{2} E(2 x ;-\mathcal{A}) \mathcal{D}_{1}\right]^{-1} E\left(\alpha+2 x ;-\mathcal{A}^{\dagger}\right) \mathcal{C}^{\dagger}
$$

where the inverse exists because of the unique solvability of (6.10). Consequently, from (4.19) and (8.2) we obtain

$$
k(x)=2 i \mathcal{C} E(2 x ;-\mathcal{A})\left[I_{p}-\mathcal{D}_{1} E\left(2 x ;-\mathcal{A}^{\dagger}\right) \mathcal{D}_{2} E(2 x ;-\mathcal{A})\right]^{-1} \mathcal{B}, \quad x>0 .
$$

With the help of (7.8), (7.10), and (7.11), we get

$$
\mathcal{D}_{1}=\left[\begin{array}{cc}
0 & 0 \\
0 & P_{1}
\end{array}\right], \quad \mathcal{D}_{2}=\left[\begin{array}{cc}
0 & 0 \\
0 & P_{2}
\end{array}\right],
$$

as well as

$$
k(x)=2 i \mathcal{C}_{+} e^{-2 x \mathcal{A}_{+}}\left[I_{p_{+}}-P_{1} e^{-2 x \mathcal{A}_{+}^{\dagger}} P_{2} e^{-2 x \mathcal{A}_{+}}\right]^{-1} \mathcal{B}_{+}, \quad x>0 .
$$

Let us now compute $k(x)$ for $x<0$, starting from $L(\lambda)$ as given in (7.47), so that

$$
\hat{L}(t)=-\Omega_{8} E\left(t ;-\Omega_{7}\right) \Omega_{9}, \quad \hat{L}(t)^{\dagger}=-\Omega_{9}^{\dagger} E\left(t ;-\Omega_{7}^{\dagger}\right) \Omega_{8}^{\dagger} .
$$

Proceeding as in the derivation of (8.3), but with $\mathcal{A}, \mathcal{B}, \mathcal{C}$, and $x$ replaced by $\Omega_{7}, \Omega_{9}, \Omega_{8}$, and $-x$, respectively, we obtain

$$
B_{r 2}(x, \alpha)=\Omega_{9}^{\dagger}\left[I_{m}-E\left(-2 x ;-\Omega_{7}^{\dagger}\right) \mathcal{D}_{4} E\left(-2 x ;-\Omega_{7}\right) \mathcal{D}_{3}\right]^{-1} E\left(\alpha-2 x ;-\Omega_{7}^{\dagger}\right) \Omega_{8}^{\dagger},
$$

where the inverse exists because of the unique solvability of (6.11). Here $\mathcal{D}_{3}$ and $\mathcal{D}_{4}$ are the positive selfadjoint matrices given by

$$
\mathcal{D}_{3}=\int_{0}^{\infty} d t E\left(t ;-\Omega_{7}\right) \Omega_{9} \Omega_{9}^{\dagger} E\left(t ;-\Omega_{7}^{\dagger}\right), \quad \mathcal{D}_{4}=\int_{0}^{\infty} d t E\left(t ;-\Omega_{7}^{\dagger}\right) \Omega_{8}^{\dagger} \Omega_{8} E\left(t ;-\Omega_{7}\right) .
$$

Using (4.19) we now obtain for $x<0$

$$
k(x)=-2 i \Omega_{9}^{\dagger}\left[I_{m}-E\left(-2 x ;-\Omega_{7}^{\dagger}\right) \mathcal{D}_{4} E\left(-2 x ;-\Omega_{7}\right) \mathcal{D}_{3}\right]^{-1} E\left(-2 x ;-\Omega_{7}^{\dagger}\right) \Omega_{8}^{\dagger},
$$


where $m=3 p$.

In order to simplify the expression for $k(x)$ in (8.4), we can use (7.48) instead of (7.47). We first replace $\left\{\Omega_{7}, \Omega_{9}, \Omega_{8}\right\}$ in (8.4) by $\{\widetilde{\mathcal{A}}, \widetilde{\mathcal{B}}, \widetilde{\mathcal{C}}\}$ and then compute the analogs $\widetilde{\mathcal{D}}_{3}$ and $\widetilde{\mathcal{D}}_{4}$ of $\mathcal{D}_{3}$ and $\mathcal{D}_{4}$. We get

$$
\widetilde{\mathcal{D}}_{3}=\left[\begin{array}{cccc}
P_{5} & 0 & 0 & 0 \\
0 & 0 & 0 & 0 \\
0 & 0 & 0 & 0 \\
0 & 0 & 0 & 0
\end{array}\right], \quad \widetilde{\mathcal{D}}_{4}=\left[\begin{array}{cccc}
P_{6} & P_{7} & 0 & 0 \\
P_{8} & P_{9} & 0 & 0 \\
0 & 0 & 0 & 0 \\
0 & 0 & 0 & 0
\end{array}\right]
$$

where

$$
P_{5}=\int_{0}^{\infty} d t e^{t \mathcal{E}_{l}^{\dagger}} \mathcal{J}_{1} \mathcal{C}^{\dagger} \mathcal{C} \mathcal{J}_{1}^{\dagger} e^{t \mathcal{E}_{l}}, \quad P_{6}=\int_{0}^{\infty} d t e^{t \mathcal{E}_{l}}\left(I_{p}+P_{3}^{\dagger} \mathcal{J}_{4}^{\dagger}\right) \mathcal{B} \mathcal{B}^{\dagger}\left(I_{p}-\mathcal{J}_{4} P_{3}\right) e^{t \mathcal{E}_{l}^{\dagger}}
$$

and $P_{7}, P_{8}$, and $P_{9}$ are irrelevant because they will not contribute to $k(x)$, as we will see. We now apply (8.4) and obtain

$$
\begin{aligned}
k(x) & =-2 i \widetilde{\mathcal{B}}^{\dagger} \Theta_{1}(x) \Theta_{2}(x) \widetilde{\mathcal{C}}^{\dagger} \\
& =-2 i \mathcal{C} \mathcal{J}_{1}^{\dagger}\left[I_{p}-e^{-2 x \mathcal{E}_{l}} P_{6} e^{-2 x \mathcal{E}_{l}^{\dagger}} P_{5}\right]^{-1} e^{-2 x \mathcal{E}_{l}}\left(I_{p}+P_{3}^{\dagger} \mathcal{J}_{4}^{\dagger}\right) \mathcal{B}, \quad x<0,
\end{aligned}
$$

where we have used (7.49)-(7.51) and

$$
\Theta_{1}(x)=\left[\begin{array}{cccc}
\mathcal{Z}_{1}(x)^{-1} & 0 & 0 & 0 \\
-\mathcal{Z}_{2}(x) \mathcal{Z}_{1}(x)^{-1} & I_{p_{-}} & 0 & 0 \\
0 & 0 & I_{p_{+}} & 0 \\
0 & 0 & 0 & I_{p}
\end{array}\right], \quad \Theta_{2}(x)=\left[\begin{array}{cccc}
e^{-2 x \mathcal{E}_{l}} & 0 & 0 & 0 \\
0 & e^{-2 x \mathcal{A}_{-}} & 0 & 0 \\
0 & 0 & 0 & 0 \\
0 & 0 & 0 & 0
\end{array}\right],
$$

with

$$
\mathcal{Z}_{1}(x)=I_{p}-e^{-2 x \mathcal{E}_{l}} P_{6} e^{-2 x \mathcal{E}_{l}^{\dagger}} P_{5}
$$

and $\mathcal{Z}_{2}(x)$ being irrelevant because it does not contribute to $k(x)$.

Now let us consider the special case where $R(\lambda)$ is analytic in $\mathbf{C}^{+}$. Then the matrix $\mathcal{A}$ has its eigenvalues in the left half plane and hence $E(t ;-\mathcal{A})=0$ for $t>0$. In that case 
$k(x)=0$ for $x>0$. We then repeat the calculations of the preceding paragraph with (7.47) replaced by $(7.34)$, i.e. with $L(\lambda)$ given by

$$
L(\lambda)=i \mathcal{B}^{\dagger}\left[\mathcal{Y} \quad I_{p}-\mathcal{Y} \mathcal{X}\right]\left(\lambda-i\left[\mathcal{A} \oplus\left(-\mathcal{E}_{l}^{\dagger}\right)\right]\right)^{-1}\left[\begin{array}{c}
\mathcal{X} \\
I_{p}
\end{array}\right] \mathcal{C}^{\dagger}
$$

In other words, we replace $\Omega_{7}, \Omega_{9}$, and $\Omega_{8}$ in $(7.34)$ by

$$
\mathcal{A} \oplus\left(-\mathcal{E}_{l}^{\dagger}\right), \quad\left[\begin{array}{c}
\mathcal{X} \\
I_{p}
\end{array}\right] \mathcal{C}^{\dagger}, \quad \mathcal{B}^{\dagger}\left[\mathcal{Y} \quad I_{p}-\mathcal{Y} \mathcal{X}\right]
$$

respectively, and employ the fact that

$$
E\left(-2 x ;(-\mathcal{A}) \oplus \mathcal{E}_{l}^{\dagger}\right)=0 \oplus E\left(-2 x ; \mathcal{E}_{l}^{\dagger}\right), \quad x<0
$$

We also replace $\mathcal{D}_{3}$ by $0 \oplus \mathcal{D}_{5}$ and $\mathcal{D}_{4}$ by $0 \oplus \mathcal{D}_{6}$, where

$$
\left\{\begin{array}{l}
\mathcal{D}_{5}=\int_{0}^{\infty} d t E\left(t ; \mathcal{E}_{l}^{\dagger}\right) \mathcal{C}^{\dagger} \mathcal{C} E\left(t ; \mathcal{E}_{l}\right) \\
\mathcal{D}_{6}=\int_{0}^{\infty} d t E\left(t ; \mathcal{E}_{l}\right)\left(I_{p}-\mathcal{X} \mathcal{Y}\right) \mathcal{B} \mathcal{B}^{\dagger}\left(I_{p}-\mathcal{Y} \mathcal{X}\right) E\left(t ; \mathcal{E}_{l}^{\dagger}\right)
\end{array}\right.
$$

As a result, we obtain from (8.4)

$$
k(x)=-2 i \mathcal{C}\left[I_{p}-E\left(-2 x ; \mathcal{E}_{l}\right) \mathcal{D}_{6} E\left(-2 x ; \mathcal{E}_{l}^{\dagger}\right) \mathcal{D}_{5}\right]^{-1} E\left(-2 x ; \mathcal{E}_{l}\right)\left(I_{p}-\mathcal{X Y}\right) \mathcal{B}, \quad x<0
$$

\section{REFERENCES}

[1] Adamyan, V. M. and Arov, D. Z.: On scattering operators and contraction semigroups in Hilbert space. Soviet Math. Dokl. 6, 1377-1380 (1965); also: Dokl. Akad. Nauk SSSR 165, 9-12 (1965) [Russian].

[2] Ablowitz, M. J. and Clarkson, P. A.: Solitons, Nonlinear Evolution Equations and Inverse Scattering. London Math. Soc. Lecture Note Series 149, Cambridge Univ. Press, Cambridge, 1991.

[3] Alpay, D. and Gohberg, I.: Inverse spectral problems for differential operators with rational scattering matrix functions. J. Differential Equations 118, 1-19 (1995).

[4] Alpay, D. and Gohberg, I.: Inverse scattering problem for differential operators with rational scattering matrix functions. In: Böttcher, A. and Gohberg, I. (Eds.): Singular Integral Operators and Related Topics (Tel Aviv, 1995), 1-18. Birkhäuser OT 90, Basel, 1996.

[5] Alpay, D. and Gohberg, I.: Potential associated with rational weights. In: Gohberg, I. and Lyubich, Yu. (Eds.): New Results in Operator Theory and its Applications, 23-40. Birkhäuser OT 98, Basel, 1997. 
[6] Alpay, D. and Gohberg, I.: State space method for inverse spectral problems. Progr. Systems Control Theory 22, 1-16 (1997).

[7] Alpay, D., Gohberg, I., and Sakhnovich, L.: Inverse scattering problem for continuous transmission lines with rational reflection coefficient function. In: Gohberg, I., Lancaster, P., and Shivakumar, P. N. (Eds.): Recent Developments in Operator Theory and its Applications (Winnipeg, MB, 1994), 1-16. Birkhäuser OT 87, Basel, 1996.

[8] Arov, D. Z.: Darlington realization of matrix-valued functions. Math. USSR Izv. 7, 1295-1326 (1973); also: Izv. Akad. Nauk SSSR Ser. Mat. 37, 1299-1331 (1973) [Russian].

[9] Bava, G. P., Ghione, G., and Maio, I.: Fast exact inversion of the generalized Zakharov-Shabat problem for rational scattering data: Application to the synthesis of optical couplers. SIAM J. Appl. Math. 48, 689-702 (1988).

[10] Beals, R. and Coifman, R. R.: Scattering and inverse scattering for first order systems. Comm. Pure Appl. Math. 37, 39-90 (1984).

[11] Beals, R. and Coifman, R. R.: Scattering and inverse scattering for first order systems: II. Inverse Problems 3, 577-593 (1987).

[12] Beals, R., Deift, P., and Tomei, C.: Direct and Inverse Scattering on the Line. Math. Surveys and Monographs, 28, Amer. Math. Soc., Providence, 1988.

[13] Bart, H., Gohberg, I., and Kaashoek, M. A.: Minimal Factorization of Matrix and Operator Functions. Birkhäuser OT 1, Basel, 1979.

[14] Bart, H., Gohberg, I., and Kaashoek, M. A.: Exponentially dichotomous operators and inverse Fourier transforms. Report 8511/M, Econometric Institute, Erasmus University of Rotterdam, The Netherlands, 1985.

[15] Bart, H., Gohberg, I., and Kaashoek, M. A.: Wiener-Hopf factorization, inverse Fourier transforms and exponentially dichotomous operators. J. Funct. Anal. 68, 1-42 (1986).

[16] Beals, R., Deift, P., and Zhou, X.: The inverse scattering transform on the line. In: Fokas, A. S. and Zakharov, V. E. (Eds.): Important Developments in Soliton Theory, 7-32. Springer, Berlin, 1993.

[17] Bhatia, R.: Matrix Analysis. Graduate Texts in Mathematics 169, Springer, New York, 1997.

[18] Böttcher, A. and Silbermann, B.: Analysis of Toeplitz operators. Springer, New York, 1990.

[19] Clancey, K. and Gohberg, I.: Factorization of Matrix Functions and Singular Integral Operators. Birkhäuser OT 3, Basel, 1981.

[20] Deift, P. and Trubowitz, E.: Inverse scattering on the line. Comm. Pure Appl. Math. 32, 121-251 (1979).

[21] Faddeev, L. D.: Properties of the $S$-matrix of the one-dimensional Schrödinger equation. Amer. Math. Soc. Transl. 2, 139-166 (1964); also: Trudy Mat. Inst. Steklova 73, 314-336 (1964) [Russian].

[22] Gasymov, M. G.: The inverse scattering problem for a system of Dirac equations of order 2n. Trans. Moscow Math. Soc. 19, 41-119 (1968); also: Trudy Moscov. Mat. Ob̌̌č. 19, 41-112 (1968) [Russian].

[23] Gohberg, I., Goldberg, S., and Kaashoek, M. A.: Classes of Linear Operators. Vol. I. Birkhäuser OT 49, Basel, 1990.

[24] Gohberg, I., Kaashoek, M. A., and Sakhnovich, A. L.: Canonical systems with rational spectral densities: Explicit formulas and applications. Math. Nachr. 149, 53-125 (1998).

[25] Gohberg, I., Kaashoek, M. A., and Sakhnovich, A. L.: Pseudocanonical systems with rational Weyl functions: Explicit formulas and applications. J. Differential Equations 146, 375-398 (1998).

[26] Gohberg, I., Kaashoek, M. A., and Sakhnovich, A. L.: Canonical systems on the line with rational spectral densities: Explicit formulas. Preprint. 
[27] Gohberg, I. and Rubinstein, S.: Proper contractions and their unitary minimal completions. In: Gohberg, I. (Ed.): Topics in Interpolation Theory of Rational Matrixvalued Functions, 223-247. Birkhäuser OT 33, Basel, 1988.

[28] Grébert, B.: Inverse scattering for the Dirac operator on the real line, Inverse Problems 8, 787-807 (1992).

[29] Hinton, D. B., Jordan, A. K., Klaus, M., and Shaw, J. K.: Inverse scattering on the line for a Dirac system. J. Math. Phys. 32, 3015-3030 (1991).

[30] Hinton, D. B. and Shaw, J. K.: Hamiltonian systems of limit point or limit circle type with both endpoints singular. J. Differential Equations 50, 444-464 (1983).

[31] Lancaster, P. and Rodman, L.: Algebraic Riccati Equations. Oxford University Press, New York, 1995.

[32] Melik-Adamyan, F. È.: On the properties of the $S$-matrix of canonical differential equations on the whole axis. Akad. Nauk Armjan. SSR Dokl. 58, 199-205 (1974) [Russian].

[33] Melik-Adamyan, F. È.: Canonical differential operators in a Hilbert space. Izv. Akad. Nauk Armjan. SSR Ser. Mat. 12, 10-31, 85 (1977) [Russian].

[34] Melik-Adamyan, F. È.: On a class of canonical differential operators. Soviet $J$. Contemporary Math. Anal. 24, 48-69 (1989); also: Izv. Akad. Nauk Armyan. SSR Ser. Mat. 24, 570-592, 620 (1989) [Russian].

[35] Petrovski, I. G.: Ordinary Differential Equations. Prentice-Hall, Englewood Cliffs, 1966.

[36] Ran, A. C. M.: Minimal factorization of selfadjoint rational matrix functions. Integral Equations Operator Theory 5, 850-869 (1982).

[37] Reed, M. and Simon, B.: Methods of Modern Mathematical Physics. III. Scattering Theory. Academic Press, New York, 1979.

[38] Sakhnovich, A. L.: Nonlinear Schrödinger equation on a semi-axis and an inverse problem associated with it. Ukr. Math. J. 42, 316-323 (1990); also: Ukrain. Mat. Zh. 42, 356-363 (1990) [Russian].

[39] Sakhnovich, L. A.: Factorization problems and operator identities. Russ. Math. Surv. 41, 1-64 (1986); also: Usp. Mat. Nauk 41, 4-55, 240 (1986) [Russian].

[40] Sakhnovich, L. A.: The method of operator identities and problems in analysis. St. Petersburg Math. J. 5, 1-69 (1994); also: Algebra i Analiz 5, 3-80 (1993) [Russian].

[41] Shabat, A. B.: An inverse scattering problem. Differential Equations 15, 1299-1307 (1980); also: Differ. Urav. 15, 1824-1834, 1918 (1979) [Russian].

AcKnowledgments. This material is based on work supported by the National Science Foundation under grants DMS-9501053 and DMS-9803219, and by C.N.R., MURST, and a University of Cagliari Coordinated Research Project. The authors are greatly indebted to André Ran for the similarity transformations $\Phi_{l}$ and $\Phi_{r}$ in Section 7 allowing them to deal with rational reflection coefficients $R(\lambda)$ having poles in $\mathbf{C}^{+}$.

$\begin{array}{ll}\text { Tuncay Aktosun } & \text { Martin Klaus } \\ \text { Dept. of Mathematics } & \text { Dept. of Mathematics } \\ \text { North Dakota State } & \text { Virginia Polytechnic Inst. } \\ \quad \text { University } & \text { and State University } \\ \text { Fargo, ND 58105 } & \text { Blacksburg, VA 24061 } \\ \text { aktosun@plains.nodak.edu } & \text { klaus@math.vt.edu }\end{array}$

MSC Primary 34A55, 81U40, Secondary 73D50

\author{
Cornelis van der Mee \\ Dipartimento di Matematica \\ Università di Cagliari \\ Via Ospedale 72 \\ 09124 Cagliari, Italy \\ cornelis@krein.unica.it
}

